Research Article

\title{
Investigation on the Nonlinear Vibration Characteristics of Current-Carrying Crescent Iced Conductors under Aerodynamic Forces, Ampere's Forces, and Forced Excitation Conditions
}

\author{
Xiaohui Liu $\mathbb{D}^{1},{ }^{1}$ Haobo Liang $\mathbb{D},{ }^{2}$ Guangyun Min $\mathbb{D D}^{3},{ }^{3}$ Chuan Wu $\mathbb{D}^{4},{ }^{4}$ and Mengqi Cai $\mathbb{D}^{5}$ \\ ${ }^{1}$ State Key Laboratory of Mountain Bridge and Tunnel Engineering, Chongqing Jiaotong University, Chongqing 400074, China \\ ${ }^{2}$ School of Civil Engineering, Chongqing Jiaotong University, Chongqing 400074, China \\ ${ }^{3}$ Sino-French Institute of Nuclear Engineering and Technology, Sun Yat-Sen University, Zhuhai Guangdong 519082, China \\ ${ }^{4}$ State Grid Henan Electric Power Research Institute, Zhengzhou 450052, China \\ ${ }^{5}$ School of Architecture and Civil Engineering, Chengdu University, Chengdu 610106, China \\ Correspondence should be addressed to Guangyun Min; guangyunmin@163.com
}

Received 10 July 2021; Revised 15 September 2021; Accepted 21 September 2021; Published 12 October 2021

Academic Editor: Sundarapandian Vaidyanathan

Copyright (C) 2021 Xiaohui Liu et al. This is an open access article distributed under the Creative Commons Attribution License, which permits unrestricted use, distribution, and reproduction in any medium, provided the original work is properly cited.

\begin{abstract}
Aiming at the problem of nonlinear vibration of current-carrying iced conductors, the aerodynamic forces are introduced into the previous vibration equation of current-carrying conductors that only considered Ampere's forces. At the same time, on this basis, a forced excitation load is further introduced to study the influence of dynamic wind on the nonlinear vibration characteristics of current-carrying iced conductors, and a new current-carrying iced conductors system under the combined action of Ampere's forces, forced excitation, and aerodynamic forces has been established, and the improved theoretical modeling of current-carrying iced transmission lines made the model more in line with practical engineering. Firstly, the model of current-carrying iced conductors was established, and then the vibration equation of the model was derived. And the vibration equation was transformed into a finite dimensional ordinary differential equation by using the Galerkin method. The amplitude-frequency response functions of the nonlinear forced primary resonances and super-harmonic and subharmonic resonances of the system are derived by using the multiscale method. Through numerical calculation, the influence of current-carrying, spacing, wind velocity, tension, and excitation amplitude on the response amplitude when the primary resonance of the system appears is analyzed, and the difference between the two working conditions (considering the aerodynamic forces and without considering aerodynamic forces) is compared. The influence of the variation of current-carrying $i$ on the response amplitude of superharmonic and subharmonic resonances and the stability of the steady-state solution of forced primary resonance was analyzed. The results show that the response amplitude and the nonlinearilty of system under the action of aerodynamic forces are smaller and weaker than without the action of aerodynamic forces; the variation of line parameters has a certain influence on the response amplitude of conductor and the nonlinearity of system; the response amplitudes of the primary resonance, super-harmonic resonance, and subharmonic resonance increase with the increase in the excitation amplitudes, and the resonance peak is offset towards the negative value of the tuning parameter $\sigma$, showing the characteristics of soft spring, and the response amplitudes are accompanied by complex nonlinear dynamic behaviors such as the multivalue and jump phenomenon. The change of currentcarrying $i$ has an obvious effect on the nonlinearity of the system. The nonlinear and response amplitudes of the system are also enhanced with the increase in wind velocity. The stability of the system is judged when the primary resonance occurs, and it is found that the response amplitude shows synchronization and the out-of-step phenomenon with the change of tuning parameters. The research results obtained in this paper would help to further improve the theoretical modeling about current-carrying iced lines, and the research of line parameters can give a certain reference value to practical engineering, and it will have a positive effect on the safe operation of high-voltage transmission lines.
\end{abstract}




\section{Introduction}

High-voltage transmission lines are an important part of power facilities; its destruction will lead to great economic losses. However, under certain meteorological conditions, the transmission lines may be covered with ice on its windward side, which makes the cross section of the transmission lines become asymmetric [1]. When the aerodynamic characteristics of the iced conductors meet certain conditions, it may produce a low-frequency, largeamplitude self-excited vibration, that is, galloping [2]. Longtime and continuous large-scale galloping of the transmission lines will cause frequent tripping and power failure of the line, metal damage, broken strands, tower damage, and other accidents, with great harm to the safe operation of the transmission lines $[3,4]$.

Therefore, in order to ensure its safe and normal operation, it is of great significance to study the galloping of the transmission lines. There are three accepted theories on the galloping mechanism of iced conductors: Den Hartog [5] and Parkinson [6] have proposed the single-degree-offreedom vertical galloping mechanism, Nigol and Buchan $[7,8]$ have proposed the two-degree-of-freedom torsional galloping mechanism, and Yu and Desai [9] have developed the three-degree-of-freedom galloping model with regard to ice eccentricity. These three kinds of galloping excitation mechanisms provide valuable theoretical basis for scholars to study the galloping of transmission lines in the future.

With the increasing development of nonlinear dynamics, scholars have noticed that the transmission lines actually belong to the flexible cable structure because the span length of the transmission lines is much larger than the diameter of the transmission lines, and they would show obvious nonlinear behavior when they are affected by gravity and external forces [10]. At the same time, since flexible cable structures have important applications in industry, automobiles, aerospace, and other structural systems, the research on the nonlinear vibration characteristics of flexible cables has become a hotspot in the past few years [11-14]. Scholars have conducted years of research on the nonlinear vibration characteristics of flexible cable structures.

Based on the research of Lee and Perkins, Italian scholar Luongo [15] creatively considered the nonnegligible geometric nonlinearity influence in the process of galloping of the transmission lines. Then, Luongo et al. [16] studied the limit motion cycle problem of the galloping response by using the multiscale method on the basis of considering the vertical and horizontal coupling 2-DOF lines galloping model with geometric nonlinearity of the structure. Based on the 3-DOF galloping mathematical model derived by $\mathrm{Yu}$, Luongo et al. [17] established a vertical, horizontal, and torsional coupling lines galloping mathematical model taking into account multiple factors such as geometric nonlinearity and dynamic torsion angle of galloping. Yan [18] used the incremental harmonic balance method to derive the motion equation of the three-degree-of-freedom galloping model of iced transmission lines and analyzed the value of the harmonic terms and the limit cycle phenomenon in the galloping process of transmission lines. Lou et al. [19] have proposed a two-parameter bifurcation analysis technique to study the bifurcation and stability of the nonlinear galloping of iced transmission lines. Yan et al. [20] studied the internal resonance phenomenon of galloping of the quad bundle conductor lines. Zhou et al. [21] studied for the first time the galloping behavior of a thin iced eight-bundle conductor transmission lines in a uniform flow field. Liu et al. [22] studied the influence of two simplified methods of dynamic tension on the galloping characteristics of transmission lines.

In fact, the span length of the transmission lines is much larger than its diameter, and the transmission lines essentially belong to cable structure, which would exhibit obvious nonlinear behavior under the action of gravity and external forces. Therefore, the academic research results on suspension cables can also bring guiding opinions for the research of transmission lines. The nonlinear dynamic behavior of cables under external excitation has always been a research hotspot. G. Rega and F. Benedettini $[23,24]$ studied the nonlinear vibration problems of elastic cables under planar excitation and super-harmonic resonance conditions, respectively. Zhao et al. [25,26] studied the nonlinear dynamic behavior of the nonlinear planar secondary resonance of suspension cables with the thermal effect and the two-to-one internal resonance of the suspension cables in thermal environments under periodic excitation, respectively. The research results of the above scholars are very meaningful. However, there are few studies on the dynamic response of cables under the unsteady wind conditions.

The findings in [5-22] have played a good role in promoting the follow-up research on the galloping of transmission lines. However, it is not difficult to find from the synthesis of their research results that these results are based on the consideration of the self-excited vibration caused by iced transmission lines under the action of stable wind, and they do not consider the influence of dynamic wind on the galloping of transmission lines. In fact, the nonlinear vibration characteristics of iced transmission lines under the action of dynamic wind are rarely studied. Zulli and Luongo $[27,28]$ considered the dynamics of two towers exposed to turbulent wind flow and linked by a nonlinear viscous device. They stated that the steady component of the wind is responsible for self-excitation, while the turbulent part causes both parametric and external excitation in a specific resonance condition. On this basis, Liu $X$ et al. [29] considered adding the external excitation load into the governing equation of iced transmission lines under the condition of stable wind and established a new forced selfexcitation system to study the influence of dynamic wind on the nonlinear galloping characteristics of iced transmission lines.

During actual transmission line operation, the high electromagnetic force may cause the adhesion accident of bundle conductor lines [30]. The phenomenon of "whipping" between subconductors has been observed in the field, which shows that the role of electromagnetic force cannot be ignored. Mehta and Swart [31] have proposed an approach for measuring the electromagnetic force between any 
number of conductors and each of arbitrary shape in space. Vasetsky [32] considered the three-dimensional electromagnetic field generated by a large cross section curved conductor and further used the formula established by the asymptotic method to calculate the three-dimensional electromagnetic force.

At the same time, the presence of the electromagnetic force indicates that there is an electromagnetic field between the transmission lines. In addition, it is necessary to study the influence of Ampere's force caused by the change of current on the nonlinear vibration characteristics of the transmission line because of the transmission lines transmission electrical power. However, the nonlinear vibration characteristics of iced transmission lines under the influence of Ampere's force are rarely studied. Xiang [33] studied the nonlinear vibration of a current-carrying conductor, but its model was too simplified. It only considered the elastic force and Ampere's force of the conductor and did not consider that the line would be affected by many environmental factors during the actual operation.

Therefore, on the basis of the above research results, this paper considers that in the actual wind field, when one current-carrying conductor in the bundle conductors is located in the electromagnetic field provided by two adjacent current-carrying conductors, it is modeled into a model composed of two long straight parallel conductors and the current-carrying conductor in the middle, and the influence of dynamic wind and Ampere's force caused by current changes on the nonlinear vibration of the conductor is considered, forming a new mathematical model of currentcarrying iced conductors under the combined action of aerodynamic force-Ampere's force-forced excitation, and the nonlinear vibration control equation is obtained, and the effects of several key parameters on the vibration characteristics of current-carrying conductors are analyzed by numerical examples, some valuable conclusions have been drawn, and it plays an active role in the safe operation of current-carrying conductors.

\section{Nonlinear Vibration Equation}

The model of the current-carrying conductors is established as shown in Figure 1. The model is exposed to the unsteady wind $U$ as a whole, uniformly acting all along their spans, where $A B$ and $C D$ are two long direct parallel currentcarrying conductors spaced $2 b$, and the current-carrying intensity is $I$; the middle one is a tension current-carrying conductor hinged at both ends, with the lengths of the span is $L$ and a current-carrying intensity of $i$, which is subjected to external harmonic excitation $F \cos (\Omega t)$ (the external excitation caused by the unsteady part of the natural wind can be simplified to this load [27-29] where $F$ is the excitation amplitude and $\Omega$ is the excitation frequency). In order to obtain the nonlinear vibration equation of the conductor, the Cartesian coordinate system $O-x-y-z$ is established with the left suspension point of the middle tension currentcarrying conductor as the origin. $O x$ axis follows the direction of the equilibrium position of the middle tension current-carrying conductor, $O y$ axis points to the long

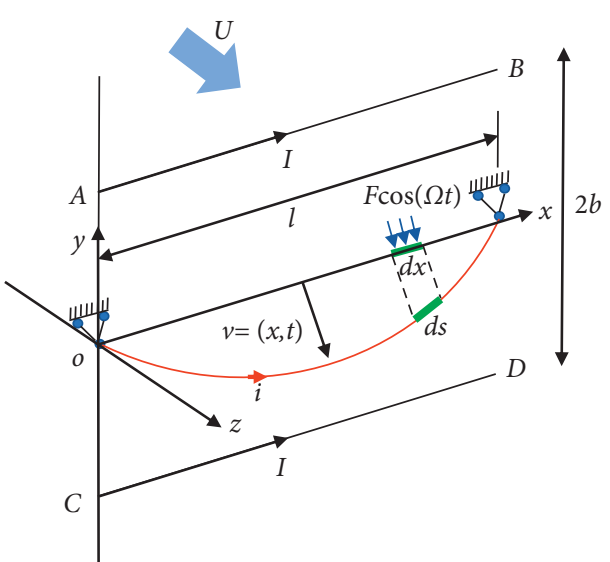

FIgURE 1: The model of current-carrying conductors.

straight parallel current-carrying conductor $A B$, and $O z$ axis is perpendicular to the plane $O x y$. The damage of the galloping of conductors to the transmission system is mainly due to the large-amplitude vibration in the plane, and the influence of vertical excitation on the system is mainly reflected in the influence of the motion in the plane. Therefore, this paper only considers the vertical vibration in the plane of the current-carrying conductor, and $v(x, t)$ is used to represent the vertical vibration displacement in the plane of the current-carrying conductor.

Taking the microelement with length of $\mathrm{d} s$ on the conductor for study as shown in Figure 2 where $T(x, t)$ is the tension of current-carrying conductor, $\alpha(x, t)$ is the angle between the microelement of the conductor and the horizontal $O x$ axis, and Ampere's force on the microelement of current $i \mathrm{~d} s$ is $\mathrm{d} f$, its direction corresponds to the vertical deviation angle $\alpha$ and can be expressed as

$$
d f=\frac{\mu I}{2 \pi}\left(\frac{1}{b-v}-\frac{1}{b+v}\right) i d s=\frac{\mu I i v d s}{\pi\left(b^{2}-v^{2}\right)},
$$

where $\mu$ is the magnetic permeability in air.

On analysis of the forces of the microelement of conductor, it is assumed that the conductor is balanced in the $x$ direction and the motion only in the $v$ direction. According to Newton's law of motion, the mechanical equilibrium equations in the $x$ direction and $v$ direction can be, respectively, expressed as

$$
\begin{aligned}
& T(x+d x, t) \cos \alpha(x+d x, t)-T(x, t) \cos \alpha(x, t) \\
& \quad-\frac{\mu I i v}{\pi\left(b^{2}-v^{2}\right)} d s \sin \alpha=0, \\
& T(x+\mathrm{d} x, t) \sin \alpha(x+\mathrm{d} x, t)-T(x, t) \sin \alpha(x, t) \\
& \quad+\frac{\mu I i u}{\pi\left(b^{2}-v^{2}\right)} \mathrm{d} s \cos \alpha=\left[\rho(x) \frac{\partial^{2} v}{\partial t^{2}}+c \frac{\partial v}{\partial t}-F \cos (\Omega t)\right. \\
& \left.-F_{\text {air }}\right] \mathrm{d} x,
\end{aligned}
$$




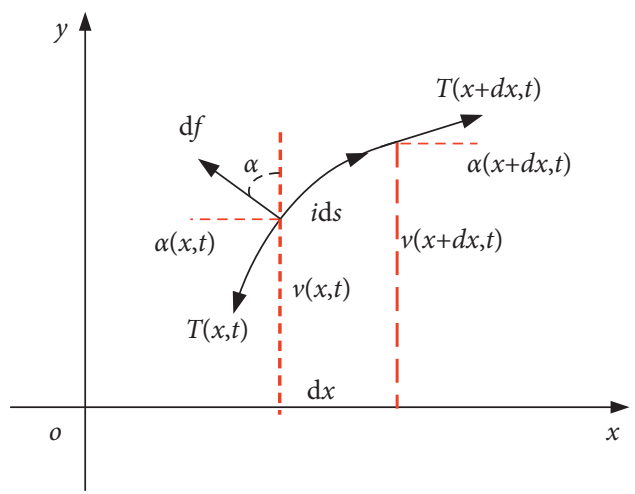

FIgURE 2: Infinitesimal force diagram of current-carrying conductors.

where $\rho(x)$ represents the linear density of the conductor, $x \epsilon$ $[0,1]$, and $c$ is the viscous damping coefficient.

Substituting $\tan \alpha=\partial v / \partial x, \quad \mathrm{~d} s \cdot \sin \alpha=\partial v / \partial x \mathrm{~d} x$, and $\mathrm{d} s \cdot \cos \alpha=\mathrm{d} x$ into equations (2) and (3), it can obtain

$$
\begin{gathered}
T(x+d x, t) \cos \alpha(x+d x, t)-T(x, t) \cos \alpha(x, t) \\
-\frac{\mu I i v}{\pi\left(b^{2}-v^{2}\right)} \frac{\partial v}{\partial x} d x=0, \\
T(x+\mathrm{d} x, t) \sin \alpha(x+\mathrm{d} x, t)-T(x, t) \sin \alpha(x, t) \\
+\frac{\mu I i v}{\pi\left(b^{2}-v^{2}\right)} \mathrm{d} x=\left[\rho(x) \frac{\partial^{2} v}{\partial t^{2}}+c \frac{\partial v}{\partial t}-F \cos (\Omega t)-F_{\text {air }}\right] \mathrm{d} x .
\end{gathered}
$$

Assuming that the density and tension of the conductor do not change with the space and time, $\rho(x)=\rho$ and $T(x, t)=$ $T$. Substituting equation (4) into (5), the following is obtained:

$$
\begin{gathered}
\frac{T}{\sqrt{1+(\partial v / \partial x)^{2}}} \frac{\partial^{2} v}{\partial x^{2}}+\frac{\mu I i v}{\pi\left(b^{2}-v^{2}\right)}\left[1+\left(\frac{\partial v}{\partial x}\right)^{2}\right] \\
=\rho \frac{\partial^{2} v}{\partial t^{2}}+c \frac{\partial v}{\partial t}-F \cos (\Omega t)-F_{\text {air }}
\end{gathered}
$$

Consider the following situation:

$$
\begin{gathered}
\frac{\partial v}{\partial x}<1, \\
\frac{v}{a}<1 .
\end{gathered}
$$

Expanding the left end of equation (6) by Taylor series, reserving it to the third term, and under the action of dynamic wind, with Ampere's force in consideration, the nonlinear vibration equation of the transverse movement of the current-carrying crescent iced conductor can obtained that

$$
\begin{gathered}
T\left[1-\frac{1}{2}\left(\frac{\partial v}{\partial x}\right)^{2}\right] \frac{\partial^{2} v}{\partial x^{2}}+\frac{\mu I i v}{\pi b^{2}}\left[1+\left(\frac{v}{b}\right)^{2}+\left(\frac{\partial v}{\partial x}\right)^{2}\right] \\
=m \frac{\partial^{2} v}{\partial t^{2}}+c \frac{\partial v}{\partial t}-F \cos (\Omega t)-F_{\text {air }} .
\end{gathered}
$$

In equation (8), $m$ is the conductor mass per unit length, $F_{\text {air }}$ is the aerodynamic load on the conductor, and it is related to the wind, which blows with a time-dependent velocity $U(t)$, and the external harmonic excitation $F \cos (\Omega t)$ can be obtained that

$$
F \cos (\Omega t)=\rho D U \sum_{i=1}^{\infty} U_{i} \cos \left(\Omega_{i} t+\phi_{i}\right) \cdot\left(C_{0}\right),
$$

where $U_{i}$ is the amplitude, $\Omega_{i}$ is the circular frequency, $\phi_{i}$ is the phase difference, $\rho$ and $D$ denote the air density and the diameter of the transmission conductor, respectively, $U$ denotes the wind velocity, and $C_{0}$ is a constant, which is related to the aerodynamic force of the iced transmission conductor.

The above partial differential equation was transformed into an ordinary differential equation by using the Galerkin method. The transverse vibration displacement $v(x, t)$ is discretized through separate variables, as follows:

$$
v(x, t)=\phi(x) \cdot q(t)
$$

where $\phi(x)=\sin (n \pi x / l)$ is modal function of iced transmission lines and $q(t)$ describes the amplitudes varying as the time $t$. In this paper, the research object is a tensioned current-carrying conductor, and its sag is very small, which shows that the quadratic nonlinearity is much weaker than the cubic one in this system, which belongs to the weak nonlinear analysis. Recalling previous discussions on the possible differences between the direct and discrete perturbation formula for the quadratic nonlinear dominated systems [34], the galloping characteristics of transmission lines are mainly studied by using the single-mode truncation in this paper. So, let $n=1$.

Substituting equations (10) into (8) and using the Galerkin discrete method lead to

$$
\vartheta_{1} \ddot{q}(t)+\vartheta_{2} \dot{q}(t)+\vartheta_{3} q(t)+\vartheta_{4} q^{3}(t)=f \cos (\Omega t)+\widetilde{F},
$$

where a dot denotes time-differentiation, and the coefficients are, respectively, expressed as follows: 


$$
\begin{aligned}
\vartheta_{1}= & -\int_{0}^{l} m \phi^{2}(x) \mathrm{d} x, \\
\vartheta_{2}= & -\int_{0}^{l} c \phi^{2}(x) \mathrm{d} x, \\
\vartheta_{3}= & \int_{0}^{l} T \phi(x) \phi^{\prime \prime}(x) \mathrm{d} x+\int_{0}^{l} \frac{\mu I i}{\pi b^{2}} \phi^{2}(x) \mathrm{d} x, \\
\vartheta_{4}= & -\int_{0}^{l} \frac{T}{2} \phi^{\prime \prime}(x) \phi^{\prime 2}(x) \phi(x) d x+\int_{0}^{l} \frac{\mu I i}{\pi b^{4}} \phi^{4}(x) d x \\
& +\int_{0}^{l} \frac{\mu I i}{\pi b^{2}} \phi^{2}(x) \phi^{\prime 2}(x) d x, \\
f= & \int_{0}^{l} F \phi(x) d x, \\
\widetilde{F}= & \int_{0}^{l} F_{\text {air }} \phi(x) d x .
\end{aligned}
$$

\section{Aerodynamic Load Model}

The iced shapes of iced transmission lines are varied, and the typical iced shapes are crescent, circular sector, and D-shaped, respectively. However, according to the classification results of the iced shapes in 124 conductor galloping observed by Japanese scholar M Masataka [35], it can be seen that crescent-shaped iced conductor occupies a considerable proportion in observations of actual conductors galloping. Therefore, the crescent iced current-carrying conductor would be taken as the research object to analyze the galloping characteristics of current-carrying conductors in this paper.

The cross section model of crescent iced conductors was established and is shown in Figure 3. Assuming the horizontal wind along the $z$ axis direction through the ice surface of conductor, the size of the real wind velocity is $U$, conductor under the action of wind load at this time will produce relative movement, leading to the traverse aerodynamic lift $F_{\mathrm{L}}$ and aerodynamic drag force $F_{\mathrm{D}}$ and horizontal $z$ axis or vertical $y$ direction angle formation, leading to the relative wind speed, and the angle formed between the real wind speed be wind attack angle $\alpha$. In Figure 3(b), $\alpha_{0}$ is the initial angle of attack, $\alpha_{\mathrm{t}}$ is the relative wind attack angle, $U_{\mathrm{r}}$ is the relative wind velocity, and $\dot{v}$ is the vertical galloping velocity.

Since this paper only studies the in-plane vertical vibration of the conductor, the aerodynamic loads on the conductor in the direction of rotation and the transverse direction can be ignored. And in the case of neglecting the aerodynamic load in the direction of rotation and transverse direction, the iced transmission line is subjected to the aerodynamic load in one direction, which is vertical direction.

According to Figure 3 and the conclusion of aerodynamics, it can obtain that

$$
F_{\text {air }}=F_{L} \cos \left(\alpha_{t}\right)-F_{D} \sin \left(\alpha_{t}\right)=0.5 \rho d U^{2} C_{y}(\alpha),
$$

where $d$ is the diameter of the conductor cross-section, $\rho$ is the density of air, and $C_{\mathrm{y}}(\alpha)$ is the aerodynamic coefficient, and based on Taylor' law, the aerodynamic coefficients in the $y$-axis direction can be fitted with a cubic nonlinear curve, and then the following equation is obtained:

$$
C_{y}(\alpha)=\eta_{1} \alpha+\eta_{2} \alpha^{2}+\eta_{3} \alpha^{3}
$$

In equation (14), $\eta_{1}, \eta_{2}$, and $\eta_{3}$ are the aerodynamic coefficients relevant to the aerodynamic load, which can be obtained through wind tunnel test [36], and the wind attack angle $\alpha$ can be expressed as follows:

$$
\alpha=\alpha_{0}+\theta-\frac{\dot{v}+1 / 2 \dot{\theta} D}{U} .
$$

Neglecting the relative wind speed angle and rotational angle caused by torsion and transforming the coordinate to the $0^{\circ}$ position of iced, the initial angle $\alpha_{0}$ is eliminated. Then, $\alpha$ is transformed into

$$
\alpha \approx-\frac{\dot{v}}{U}
$$

By combining equations (13), (14), and (16), the following can be obtained:

$$
F_{\text {air }}=\frac{1}{2} \rho U^{2} D\left[\eta_{1}\left(-\frac{\dot{v}}{U}\right)+\eta_{2}\left(-\frac{\dot{v}}{U}\right)^{2}+\eta_{3}\left(-\frac{\dot{v}}{U}\right)^{3}\right] .
$$

By substituting equations (13) and (17) into (11), the nonlinear vibration equation of current-carrying iced conductor considering aerodynamic load can be obtained:

$$
\begin{aligned}
& \vartheta_{1} \ddot{q}(t)+\left(\vartheta_{2}+\widetilde{\vartheta}_{2}\right) \dot{q}(t)+\vartheta_{3} q(t)+\vartheta_{4} q^{3}(t)+\vartheta_{5} \dot{q}^{2}(t) \\
& \quad+\vartheta_{6} \dot{q}^{3}(t)+f \cos (\Omega t)=0,
\end{aligned}
$$

where

$$
\begin{aligned}
& \widetilde{\vartheta}_{2}=-\int_{0}^{l} \frac{U}{2} \rho d \eta_{1} \phi^{2}(x) \mathrm{d} x, \\
& \vartheta_{5}=\int_{0}^{l} \frac{1}{2} \rho d \eta_{2} \phi^{3}(x) \mathrm{d} x, \\
& \vartheta_{6}=-\int_{0}^{l} \frac{1}{2 U} \rho d \eta_{3} \phi^{4}(x) \mathrm{d} x .
\end{aligned}
$$

\section{Nonlinear Dynamic Response: Primary Resonance}

In order to further discuss and analyze the nonlinear vibration characteristics of the current-carrying iced conductor, the multiscale method $[37,38]$ was used to analyze and solve the dynamic response of the conductors. Based on the basic idea of the multiscale method, the dimensionless small quantity $\varepsilon$ is introduced. 


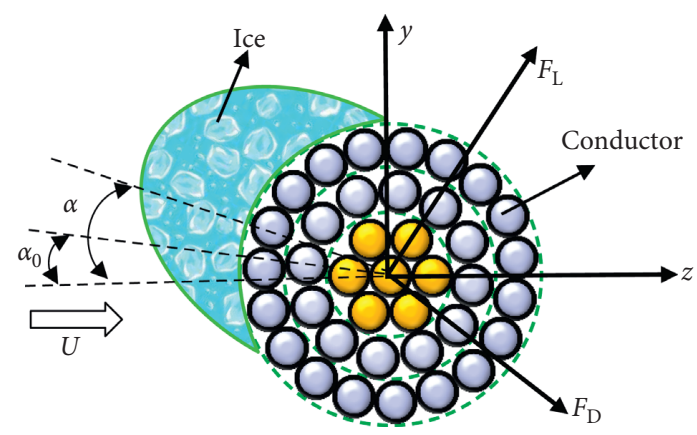

(a)

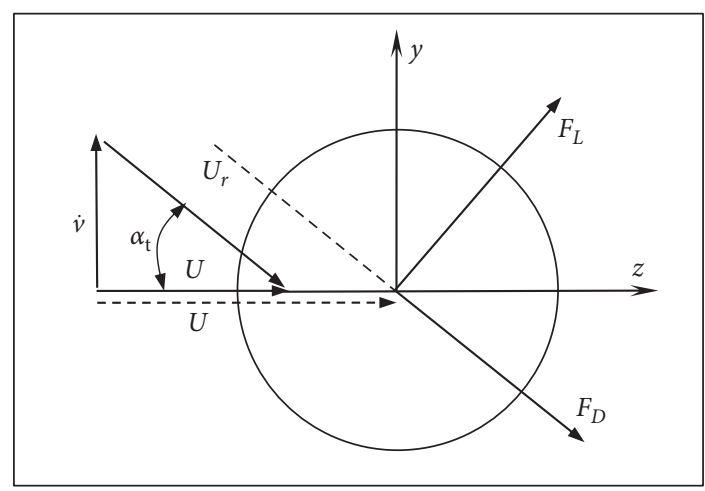

(b)

Figure 3: (a) The cross sectional model of crescent iced conductor. (b) Relative flow in the quasi-steady assumption.

Meanwhile, in order to make the damping effect, nonlinearity, and excitation all appear in the same order, the form of equation (18) needs to be transformed into

$$
\begin{aligned}
\ddot{q}(t)+\omega_{0}^{2} q(t)= & \varepsilon\left(\vartheta_{k} \dot{q}(t)+\vartheta_{m} q^{3}(t)+\vartheta_{n} \dot{q}^{2}(t)+\vartheta_{v} \dot{q}^{3}(t)\right) \\
& +\varepsilon P \cos (\Omega t),
\end{aligned}
$$

where $\varepsilon<<1$, and the new linear and nonlinear coefficients in equation (20) are expressed as follows:

$$
\begin{gathered}
\omega_{0}^{2}=\frac{\vartheta_{3}}{\vartheta_{1}} ; \\
\vartheta_{k}=-\frac{\vartheta_{2}}{\vartheta_{1}} ; \\
\vartheta_{m}=-\frac{\vartheta_{4}}{\vartheta_{1}} ; \\
\vartheta_{n}=-\frac{\vartheta_{5}}{\vartheta_{1}} ; \\
P=\frac{f}{\vartheta_{1}},
\end{gathered}
$$

where $\omega_{0}$ represents the frequency in the vertical direction.

Set the first-order approximate solution of the primary resonance as follows:

$$
q(t)=q_{0}\left(T_{0}, T_{1}\right)+\varepsilon q_{1}\left(T_{0}, T_{1}\right)+\mathrm{O}\left(\varepsilon^{2}\right) .
$$

In equation (22), $q_{0}$ is the vibration displacement, $q 1$ is the modified value of the vibration displacement, $\mathrm{O}\left(\varepsilon^{2}\right)$ represents the higher-order term, and $T_{0}$ and $T_{1}$ represent two different time scales, respectively, and satisfy the following relation:

$$
\begin{aligned}
& T_{0}=t ; \\
& T_{1}=\varepsilon t .
\end{aligned}
$$

Then,

$$
\frac{d}{d t}=\frac{d T_{0}}{d t} \frac{\partial}{\partial T_{0}}+\frac{d T_{1}}{d t} \frac{\partial}{\partial T_{1}}+\cdots=D_{0}+\varepsilon D_{1}+\cdots
$$

$$
\frac{d^{2}}{d t^{2}}=\frac{\partial^{2}}{\partial T_{0}^{2}}+2 \varepsilon \frac{\partial}{\partial T_{0} \partial T_{1}}+\cdots=D_{0}^{2}+2 \varepsilon D_{0} D_{1}+\cdots
$$

Introduced resonance relation is as follows: $\Omega=\omega+\varepsilon \sigma$, where $\sigma$ is the tuning parameter.

By combining equations (22)-(24) with (20) and comparing the coefficients of $\varepsilon$ in the same power term, the following equations can be obtained:

Order-\&epsi;0 is as follows:

$$
D_{0}^{2} q_{0}+\omega_{0}^{2} q_{0}=0 .
$$

Order- $\varepsilon 1-$ is as follows:

$$
\begin{aligned}
D_{0}^{2} q_{1}+\omega_{0}^{2} q_{1}= & -2 D_{0} D_{1} q_{0}+\vartheta_{k} D_{0} q_{0}+\vartheta_{m} q_{0}^{3}+\vartheta_{n}\left(D_{0} q_{0}\right)^{2} \\
& +\vartheta_{v}\left(D_{0} q_{0}\right)^{3}+P \cos \left(\omega_{0} T_{0}+\sigma T_{1}\right) .
\end{aligned}
$$

The solution of equation (25) can be expressed as

$$
q_{0}=A\left(T_{1}\right) \exp \left(i \omega_{0} T_{0}\right)+c c,
$$

where $\mathrm{cc}$ represents the complex conjugation, $i$ is the imaginary unit, and $A$ is unknown complex amplitudes. $A$ can be written in polar form: $A=a(t) \exp (\mathrm{i} \beta) / 2$, where $a$ is the amplitude of galloping and $\beta$ is the phase of galloping.

Substitute equation (27) into (26) to obtain

$$
\begin{aligned}
D_{0}^{2} q_{1}+\omega_{0}^{2} q_{1}= & \left(\vartheta_{\mathrm{m}} A^{3}-\vartheta_{\mathrm{v}} i \omega_{0}^{3} A^{3}\right) \exp \left(3 i \omega_{0} T_{0}\right) \\
& -\vartheta_{\mathrm{n}} \omega_{0}^{2} A^{2} \exp \left(2 i \omega_{0} T_{0}\right)+\vartheta_{\mathrm{n}} \omega_{0}^{2} A \bar{A} \\
& +\left(-2 i \omega_{0} A^{\prime}+\vartheta_{\mathrm{k}} i \omega_{0} A+3 \vartheta_{\mathrm{m}} A^{2} \bar{A}\right. \\
& \left.+3 \vartheta_{\mathrm{v}} i \omega_{0}^{3} A^{2} \bar{A}\right) \exp \left(i \omega_{0} T_{0}\right) \\
& +\frac{P}{2} \exp \left(i \omega_{0} T_{0}\right) \exp \left(i \sigma T_{1}\right)+c c .
\end{aligned}
$$


Eliminating the secular terms of equation (28),

$$
-2 i \omega_{0} A^{\prime}+\vartheta_{k} i \omega_{0} A+3 \vartheta_{m} A^{2} \bar{A}+3 \vartheta_{v} i \omega_{0}^{3} A^{2} \bar{A}+\frac{P}{2} \exp \left(i \sigma T_{1}\right)=0 .
$$

Substituting $A$ into equation (29) and separating the real part and imaginary part, the following can be obtained:

$$
\begin{aligned}
D_{1} a & =\frac{1}{2} a \vartheta_{\mathrm{k}}+\frac{3}{8} \vartheta_{\mathrm{v}} \omega_{0}^{2} a^{3}+\frac{P}{2 \omega_{0}} \sin \left(\sigma T_{1}-\beta\right), \\
a D_{1} \beta & =-\frac{3}{8 \omega_{0}} \vartheta_{\mathrm{m}} a^{3}-\frac{P}{2 \omega_{0}} \cos \left(\sigma T_{1}-\beta\right) .
\end{aligned}
$$

By introducing $\varphi=\sigma T 1-\beta$, equations (30a) and (30b) can be transformed into

$$
\begin{gathered}
D_{1} a=\frac{a \vartheta_{k}}{2}+\frac{3 \vartheta_{v} \omega_{0}^{2} a^{3}}{8}+\frac{P}{2 \omega_{0}} \sin \varphi, \\
a D_{1} \varphi=\sigma a+\frac{3 \vartheta_{m} a^{3}}{8 \omega_{0}}-\frac{P}{2 \omega_{0}} \cos \varphi .
\end{gathered}
$$

When $\mathrm{D} 1 a=\mathrm{D} 1 \varphi=0$, there is a steady-state motion in the system. The amplitude-frequency response equation can be obtained by squaring and adding two equations as

$$
\left(\sigma a+\frac{3 \vartheta_{\mathrm{m}} a^{3}}{8 \omega_{0}}\right)^{2}+\left(\frac{a \vartheta_{\mathrm{k}}}{2}+\frac{3 \vartheta_{\mathrm{v}} \omega_{0}^{2} a^{3}}{8}\right)^{2}=\frac{P^{2}}{4 \omega_{0}^{2}} .
$$

When the influence of aerodynamic force is not considered, the amplitude-frequency response equation of the conductor can be obtained from equation (20), which can be written as follows:

$$
\left(\sigma a+\frac{3 \vartheta_{m} a^{3}}{8 \omega_{0}}\right)^{2}+\left(\frac{a \widetilde{\vartheta}_{k}}{2}\right)^{2}=\frac{f^{2}}{4 \omega_{0}^{2}}
$$

where

$$
\begin{aligned}
& \widetilde{\vartheta}_{k}=\frac{\widetilde{\vartheta}_{2}}{\vartheta_{1}} \\
& \widetilde{\vartheta}_{2}=-\int_{0}^{l} c \phi^{2}(x) d x .
\end{aligned}
$$

\section{Nonlinear Dynamic Response: Harmonic Resonance}

This section continues to study the nonlinear vibration characteristics of current-carrying iced conductors under external harmonic excitations but focuses on the possible resonance of the system when the excitation frequency is far from the natural frequency of the system, which is referred to as the secondary resonance [25]. Since the excitation frequency $\Omega$ is far away from $\omega 0$, the excitation amplitude is no longer required to be small, but the nonlinearity and damping of the system are still limited to be relatively weak. The small parameter $\varepsilon$ before the forced excitation term in equation (20) is eliminated, and the remaining parameters remain unchanged, which can be transformed into forced vibration under strong excitation as

$$
\begin{aligned}
\ddot{q}(t)+\omega_{0}^{2} q(t)= & \varepsilon\left(\vartheta_{\mathrm{k}} \dot{q}(t)+\vartheta_{\mathrm{m}} q^{3}(t)+\vartheta_{\mathrm{n}} \dot{q}^{2}(t)+\vartheta_{\mathrm{v}} \dot{q}^{3}(t)\right) \\
& +P \cos (\Omega t) .
\end{aligned}
$$

The multiscale method is also used to solve the equation. The solutions of $q$ can be set as

$$
q(t)=q_{0}\left(T_{0}, T_{1}\right)+\varepsilon q_{1}\left(T_{0}, T_{1}\right) .
$$

Substituting equations (36) into (35) and comparing the coefficients of $\varepsilon$ in the same power term, the following equations can be obtained:

Order-¿repsi;0 is as follows:

$$
D_{0}^{2} q_{0}+\omega_{0}^{2} q_{0}=P \cos \left(\Omega T_{0}\right)
$$

Order- $\varepsilon 1$ is as follows:

$$
\begin{aligned}
D_{0}^{2} q_{1}+\omega_{0}^{2} q_{1}= & -2 D_{0} D_{1} q_{0}+\vartheta_{\mathrm{k}} D_{0} q_{0}+\vartheta_{\mathrm{m}} q_{0}^{3} \\
& +\vartheta_{\mathrm{n}}\left(D_{0} q_{0}\right)^{2}+\vartheta_{\mathrm{v}}\left(D_{0} q_{0}\right)^{3} .
\end{aligned}
$$

The solution of equation (37) is

$$
q_{0}=A\left(T_{1}\right) \exp \left(\mathrm{i} \omega_{0} T_{0}\right)+B \exp \left(\mathrm{i} \Omega T_{0}\right)+c c,
$$

where

$$
\begin{aligned}
& A=\frac{1}{2} a(t) \exp (i \beta), \\
& B=\frac{P}{\left|\omega_{0}^{2}-\Omega^{2}\right|} .
\end{aligned}
$$



that

Substituting equations (39) into (38), it can be obtained

$$
\begin{aligned}
D_{0}^{2} q_{1}+\omega_{0}^{2} q_{1}= & -2 i \omega_{0} D_{1} A \exp \left(i \omega_{0} T_{0}\right)+\vartheta_{\mathrm{k}} A i \omega_{0} \exp \left(i \omega_{0} T_{0}\right) \\
& +\vartheta_{\mathrm{k}} B i \Omega \exp \left(i \Omega T_{0}\right)+\vartheta_{\mathrm{m}}\left[A^{3} \exp \left(3 i \omega_{0} T_{0}\right)+B^{3} \exp \left(3 i \Omega T_{0}\right)+6 A \bar{A} B \exp \left(i \Omega T_{0}\right)+3 \bar{A}^{2} B \exp i\left(\Omega-2 \omega_{0}\right) T_{0}\right. \\
& \left.+6 B A \bar{B} \exp \left(i \omega_{0} T_{0}\right)+3 \bar{A} A^{2} \exp \left(i \omega_{0} T_{0}\right)+3 \bar{B} B^{2} \exp \left(i \Omega T_{0}\right)\right] \\
& +\vartheta_{\mathrm{n}}\left[-A^{2} \omega_{0}^{2} \exp \left(2 i \omega_{0} T_{0}\right)+2 A \bar{A} \omega_{0}^{2}-B^{2} \Omega^{2} \exp \left(2 i \Omega T_{0}\right)+2 B \bar{A} \omega_{0} \Omega \exp i\left(\Omega-\omega_{0}\right) T_{0}\right] \\
+ & \vartheta_{\mathrm{v}}\left[-3 i \bar{A}^{2} \omega_{0}^{2} \Omega B \exp i\left(\Omega-2 \omega_{0}\right) T_{0}-6 A i \bar{A} \omega_{0}^{2} \Omega \bar{B} \exp \left(i \Omega T_{0}\right)\right. \\
+ & 6 A i \bar{A} \omega_{0}^{2} \Omega B \exp \left(i \Omega T_{0}\right)+3 i \bar{A} A^{2} \omega_{0}^{3} \exp \left(i \omega_{0} T_{0}\right)+6 A i \omega_{0} \Omega^{2} \bar{B} B \\
& \left.\exp \left(i \omega_{0} T_{0}\right)-B^{3} \Omega^{3} i \exp \left(3 i \Omega T_{0}\right)+3 B^{2} \Omega^{3} i \bar{B} \exp \left(i \Omega T_{0}\right)\right]+c c
\end{aligned}
$$

According to the right end of the above equation, it can be found that when the excitation frequency $\Omega^{\sim} 2 \omega 0,3 \omega 0,1 /$ $2 \omega 0$, and $1 / 3 \omega 0$, the system will produce the $1 / 2$-order and $1 /$ 3 -order subharmonic resonances and 2-order and 3-order super-harmonic resonances, respectively.

5.1. 1/2-Order Subharmonic Resonance. In order to study the $1 / 2$-order subharmonic resonance of the system, a new excitation frequency misalignment quantity $\sigma$ is defined to make $\Omega=2 \omega_{0}+\varepsilon \sigma$. Substituting $\Omega$ into equation (42), eliminating the secular terms, and separating the real parts and imaginary parts, the amplitude-frequency response equation of $1 / 2$-order subharmonic resonance can be obtained by using the method of solving the primary resonance:

$$
\begin{aligned}
& \left(\sigma a+\frac{a P^{2} \vartheta_{\mathrm{m}}}{12 \omega_{0}^{5}}+\frac{3 \vartheta_{\mathrm{m}} a^{3}}{8 \omega_{0}}\right)^{2}+\left(\frac{a \vartheta_{\mathrm{k}}}{2}+\frac{3 \vartheta_{\mathrm{v}} \omega_{0}^{2} a^{3}}{8}+\frac{a P^{2} \vartheta_{\mathrm{v}}}{3 \omega_{0}^{2}}\right)^{2} \\
& =\left(\frac{a \vartheta_{\mathrm{n}} P}{3 \omega_{0}}\right)^{2} .
\end{aligned}
$$

5.2. 1/3-Order Subharmonic Resonance. So similarly, when the excitation frequency $\Omega=3 \omega 0+\varepsilon \sigma$, the system will produce $1 / 3$-order subharmonic resonance. Substituting $\Omega$ into equation (42), the amplitude-frequency response equation of 1/3-order subharmonic resonance can be obtained:

$$
\begin{aligned}
& \left(\frac{\sigma a}{3}+\frac{3 a P^{2} \vartheta_{\mathrm{m}}}{256 \omega_{0}^{5}}+\frac{3 \vartheta_{\mathrm{m}} a^{3}}{8 \omega_{0}}\right)^{2}+\left(\frac{a \vartheta_{\mathrm{k}}}{2}+\frac{3 \vartheta_{\mathrm{v}} \omega_{0}^{2} a^{3}}{8}+\frac{27 a P^{2} \vartheta_{\mathrm{v}}}{256}\right)^{2} \\
& =\frac{9 P^{2} a^{4}}{4096}\left(\frac{\vartheta_{\mathrm{m}}^{2}}{\omega_{0}}+81 \omega_{0}^{2} \vartheta_{\mathrm{v}}^{2}\right) .
\end{aligned}
$$

5.3. 2-Order Super-Harmonic Resonance. In order to study the 2-order super-harmonic resonance of the system, the excitation frequency $\Omega$ is defined as $1 / 2$ times of the natural frequency $\omega 0$; let $2 \Omega=\omega_{0}+\varepsilon \sigma$. Substituting it into equation (42), the amplitude-frequency response equation of 2 -order super-harmonic resonance can be obtained:

$$
\begin{aligned}
& \left(\sigma a+\frac{4 a P^{2} \vartheta_{\mathrm{m}}}{3 \omega_{0}^{5}}+\frac{3 \vartheta_{\mathrm{m}} a^{3}}{8 \omega_{0}}\right)^{2}+\left(\frac{a \vartheta_{\mathrm{k}}}{2}+\frac{3 \vartheta_{\mathrm{v}} \omega_{0}^{2} a^{3}}{8}+\frac{a P^{2} \vartheta_{\mathrm{v}}}{3 \omega_{0}^{2}}\right)^{2} \\
& =\left(\frac{\vartheta_{\mathrm{n}} P^{2}}{9 \omega_{0}^{3}}\right)^{2} .
\end{aligned}
$$

5.4. 3-Order Super-Harmonic Resonance. So similarly, let $3 \Omega=\omega_{0}+\varepsilon \sigma$. Substituting it into equation (42), the amplitude-frequency response equation of 3-order super-harmonic resonance can be obtained:

$$
\begin{gathered}
\left(\sigma a+\frac{243 a P^{2} \vartheta_{m}}{256 \omega_{0}^{5}}+\frac{3 \vartheta_{m} a^{3}}{8 \omega_{0}}\right)^{2}+\left(\frac{a \vartheta_{k}}{2}+\frac{3 \vartheta_{v} \omega_{0}^{2} a^{3}}{8}+\frac{27 a P^{2} \vartheta_{v}}{256}\right)^{2} \\
=\left(\frac{27 P^{3} \vartheta_{v}}{4096 \omega_{0}^{4}}\right)^{2}+\left(\frac{729 P^{3} \vartheta_{m}}{4096 \omega_{0}^{7}}\right)^{2} .
\end{gathered}
$$

\section{Numerical Example Analysis}

In this section, the primary resonance and harmonic resonance of the current-carrying iced conductors are numerically analyzed and the influences of different parameters on the nonlinear vibration characteristics of current-carrying iced conductors are discussed, and the frequencyamplitude response curves are drawn by Maple as shown in Figures 4-20. In these figures, the stability analysis of the steady-state solutions has been studied. If the partial derivative $(\partial W(\sigma, a, p) / \partial a)$ of the curve is positive, it means that the curve is stable; if the partial derivative $(\partial W(\sigma, a, p) /$ 
$\partial a)$ of the curve is negative, it means that the curve is unstable. And the solid lines represent the stable steady-state solutions, and the dashed lines represent the unstable steadystate solutions [27].

If no special statement is made, the physical parameters of current-carrying iced conductor in this paper are as follows: $l=50 \mathrm{~m}, \quad m=1.53 \mathrm{~kg} / \mathrm{m}, d=0.01880 \mathrm{~m}, c=0.006$, $T=50 \mathrm{~N}, \quad b=0.5 \mathrm{~m}, \quad \mu=4 \pi \times 10^{-7} \mathrm{H} / \mathrm{m}, \quad i=30 \mathrm{~A}, \quad I=30 \mathrm{~A}$, $U=4 \mathrm{~m} / \mathrm{s}, \eta_{1}=-0.6670, \eta_{2}=-16.2188$, and $\eta_{3}=33.4324$. The aerodynamic coefficients $\eta_{1}, \eta_{2}$, and $\eta_{3}$ are taken from [38] for the crescent iced single conductor.

6.1. Primary Resonance. Because this paper further considers Ampere's force on the basis of previous studies on the nonlinear vibration of conductors, it is necessary to make a detailed analysis of the influence of current-carrying $i$ on the nonlinear vibration characteristics of the current-carrying iced conductors. By observing equations (12a) and (12b), it can be seen that the current-carrying $i$ and direct current $I$ always appear in the form of the product, so this paper only analyzes the influence of the change of current-carrying $i$ on the response amplitude of the system. Substituting the physical parameters of the current-carrying iced conductor given above into equation (32), the amplitude-frequency response curve varying with the carrying current $i$ can be drawn by the mathematical software Maple, as shown in Figure 4.

As shown in Figure 4 (the tuning parameter $\sigma$ on the abscissa is dimensionless, the unit of excitation amplitude $P$ on the abscissa is $\mathrm{N} / \mathrm{m}$, and the actual unit of amplitude $a$ on the ordinate is $m$; the definition of the unit of tuning parameter $\sigma$ and excitation amplitude $P$ on the abscissa and response amplitude $a$ on the ordinate in other figures is consistent with Figure 4), regardless of whether considering the aerodynamic force, the response amplitude decreases with the increase in the current-carrying $i$, respectively; by $i_{5}$ falling to $i_{1}$ and $i_{55}$ falling to $i_{11}$ (data are shown in Table 1, in which the tuning parameter $\sigma=0$ and the excitation amplitude $P=0.02916 \mathrm{~N} / \mathrm{m}$ ), the curves bending to the left show the soft spring characteristics. And the degree of curve bending to the left is getting bigger and bigger as the currentcarrying $i$ increases constantly and the response amplitude with the phenomenon of jump and multivalue shows obvious nonlinear characteristics. By comparing Figures 4(a) and 4(b), it can be found that in both cases that the amplitude-frequency curve with the variation of current-carrying $i$ generally presents the same rule. The main difference is that the bending degree of amplitude-frequency curve without considering the aerodynamic forces is greater than that the bending degree of amplitude-frequency curve with considering the aerodynamic forces, which indicates that the nonlinearity of the system without considering the aerodynamic forces is much stronger. At the same time, combining with the data in Table 1, it is not difficult to find that the response amplitude with the considering aerodynamic forces is smaller than that without considering the aerodynamic forces. This is because that the system adds nonlinear damping when considering aerodynamic forces. In other words, the aerodynamic forces increase the damping energy dissipation of the system. Therefore, the response amplitude of the system will definitely be reduced compared with the case where the aerodynamic forces are not considered.

In order to further analyze the influence of currentcarrying $i$ on the primary resonance response of the system, the excitation amplitude $(p)$-response amplitude $(a)$ curve is drawn by Maple, where the tuning parameter $\sigma=-0.1$, as shown in Figure 4(c).

It can be found by observing Figure 4(c) that when the tuning parameter $\sigma=-0.1$, as the excitation amplitude $P$ increases, the response amplitude also increases and accompanies by jump and multivalue phenomena, but this phenomenon is mainly concentrated in the range of the excitation amplitude $P=0-0.1 \mathrm{~N} / \mathrm{m}$; when the excitation amplitude continues to increase to 0.2 or even greater, the growth curve of the response amplitude becomes gentle. When the current-carrying $i=30 \mathrm{~A}$, the response amplitude increases rapidly between $P=0-0.2 \mathrm{~N} / \mathrm{m}$, but there is no jump and multivalue phenomenon. When the excitation amplitude $P$ continues to increase to $0.6 \mathrm{~N} / \mathrm{m}$, the increasing of response amplitude has become relatively modest. As the current-carrying $i$ increases from $180 \mathrm{~A}$ to $780 \mathrm{~A}$, the response amplitude shows the jump and multivalue phenomenon under a small excitation amplitude, indicating that the nonlinearity of the system becomes stronger with the increase in the current-carrying $i$. When the excitation amplitude $P>0.2 \mathrm{~N} / \mathrm{m}$, the response amplitude decreases with the increase in the current-carrying $i$.

In order to analyze the influence of spacing $b$ on the primary resonance of current-carrying iced conductors, the frequency-amplitude curve of equation (32) is drawn by Maple as shown in Figure 5, in which the excitation amplitude $P=0.02916 \mathrm{~N} / \mathrm{m}$.

As shown in Figure 5(a), when the influence of aerodynamic force is taken into account, the resonance amplitude increases from $b_{11}$ to $b_{55}$, as the spacing $b$ increases (see Table 2 for the data of each point, in which tuning parameter $\sigma=0$ ), and the amplitude-frequency response curve of the system gradually changes from bending to vertical, which indicates that the nonlinearity of the system is gradually weakened. When the spacing $b$ is less than $0.3 \mathrm{~m}$, the system shows strong nonlinear characteristics, and the response amplitude is accompanied by obvious jump and multivalue phenomena. These phenomena show that the smaller the spacing between conductors, the more obvious the influence of the electromagnetic field. Therefore, in practical engineering, the spacing of split conductors needs to be carefully considered. By comparing Figures 5(a) and 5(b), it can be found that the bending degree of amplitude-frequency curve without considering aerodynamic forces is greater than that considering aerodynamic forces, which indicates that the nonlinearity of the system without considering aerodynamic forces is much stronger. This is consistent with the conclusion obtained from the amplitude-frequency curve varying with the carrying current $i$.

In order to further analyze the influence of spacing $b$ on the primary resonance of the system, the excitation amplitude $(p)$-response amplitude $(a)$ curve is drawn by Maple as shown in Figure 5(c). 


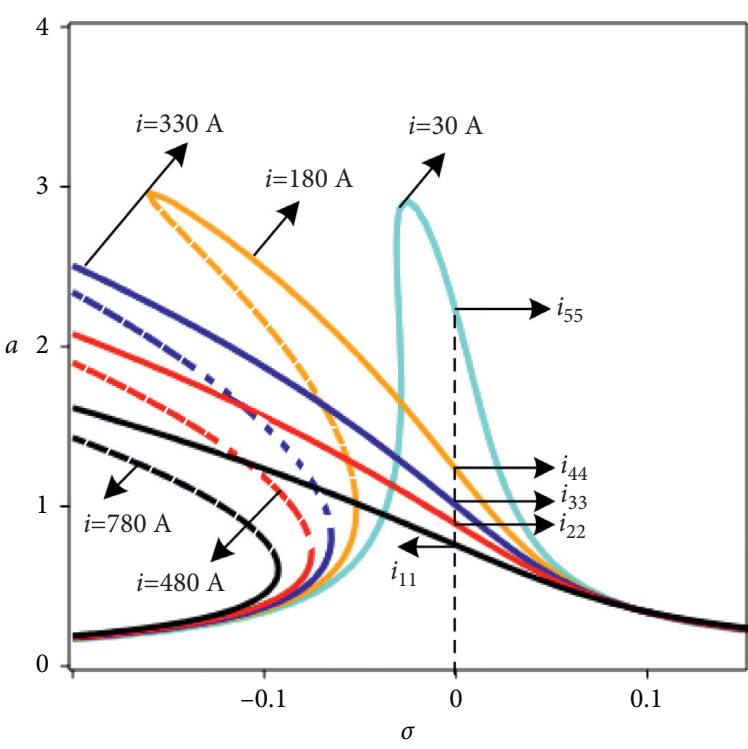

- Stable
- - Unstable

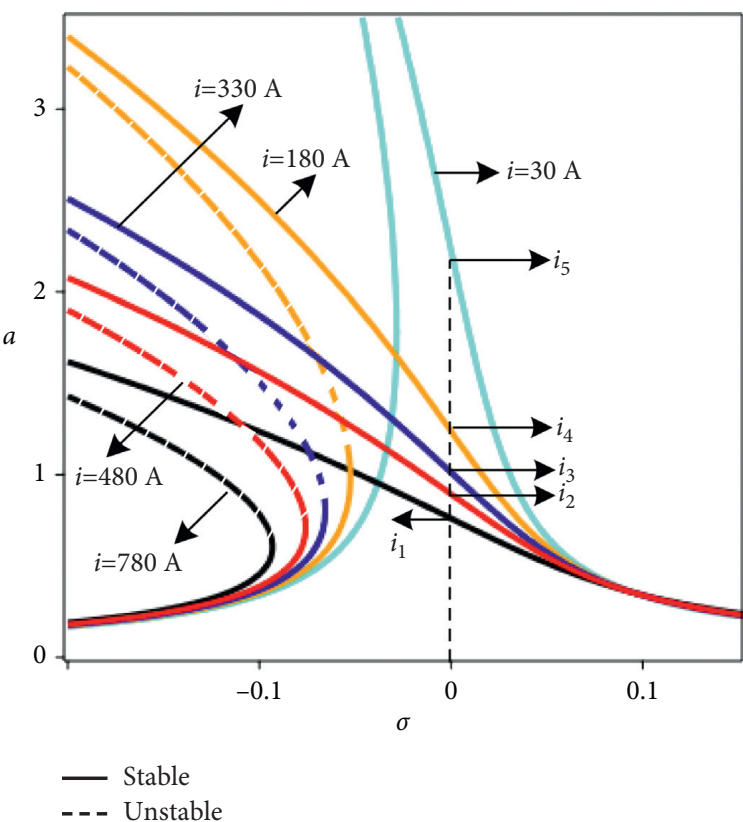

(b)

(a)

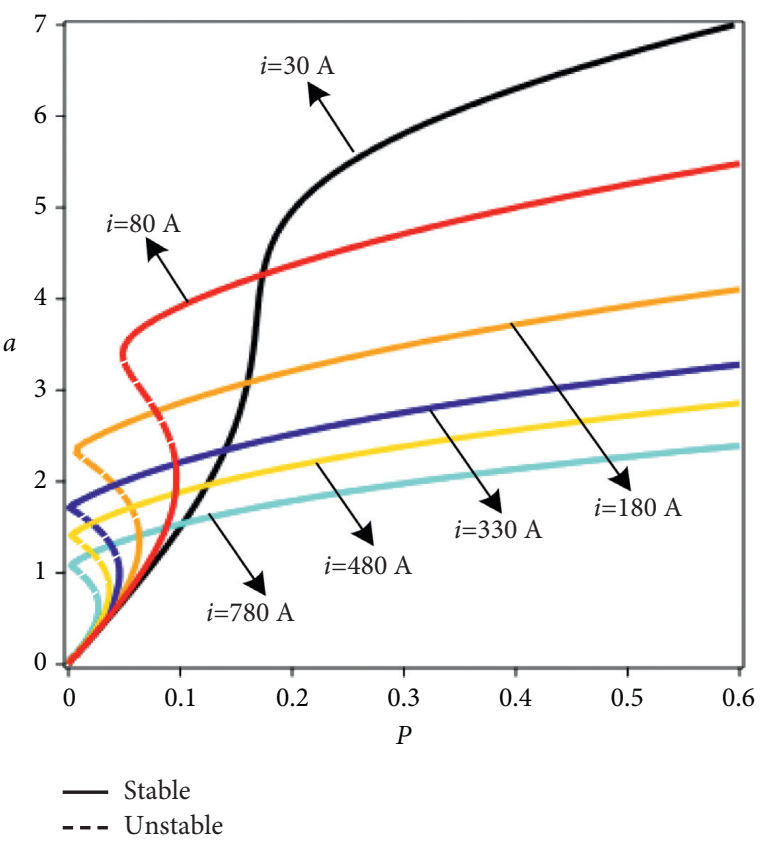

(c)

FIGURE 4: The amplitude-frequency response curve of primary resonance (varied (i)) (a) considering aerodynamic force; (b) without considering aerodynamic force; (c) with different excitation amplitudes $(\sigma=-0.1)$.

Figure 5(c) compares the nonlinear vibration characteristics of response amplitude of the primary resonance under different spacings, in which the tuning parameter $\sigma$ is -0.1 . Observing Figure 5(c), it can be seen that when the spacing $b<0.4 \mathrm{~m}$, as the excitation amplitude $P$ increases from 0 to $0.15 \mathrm{~N} / \mathrm{m}$, the response amplitude increases faster and is accompanied by jumps and multivalue phenomena. The excitation amplitude $P$ continues to increase, and the increase in response amplitude gradually becomes flat. When the spacing $b=0.5 \mathrm{~m}$ and $0.6 \mathrm{~m}$, the response amplitude also increases with the increase in the excitation amplitude $P$, but there is no jump and multivalue phenomenon. When the excitation amplitude $P>0.5 \mathrm{~N} / \mathrm{m}$, the response amplitude increases as the spacing $b$ increases. Moreover, with the decrease in wind velocity, it is easier to generate the jump phenomenon of response amplitude with a small excitation amplitude, indicating that the nonlinearity of the system increases with the decrease in the spacing $b$.

In order to verify the accuracy of the approximate solution of the frequency-amplitude equation, the same 


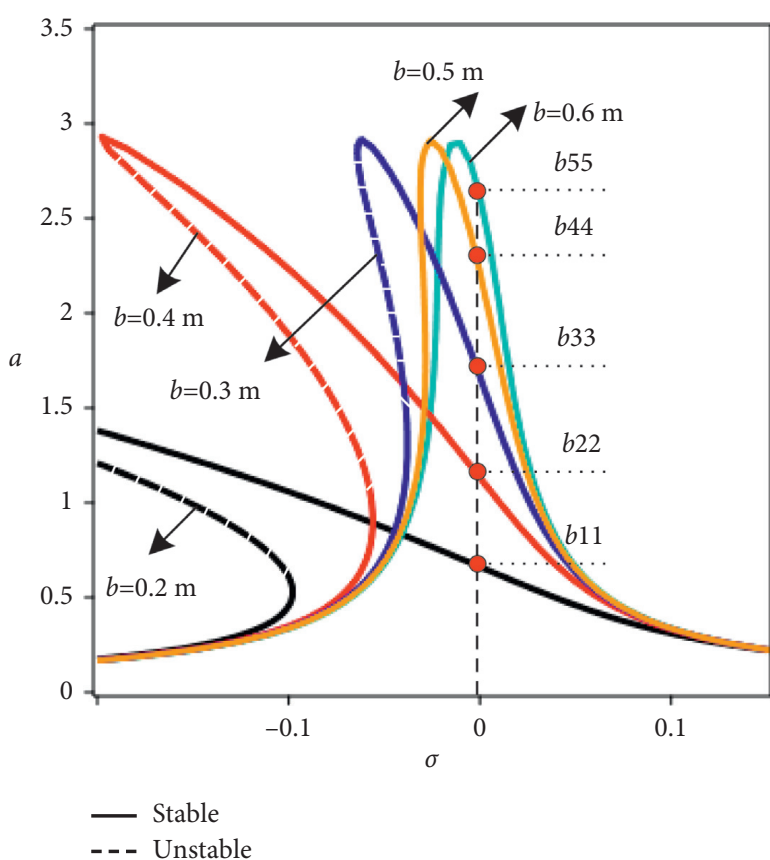

(a)

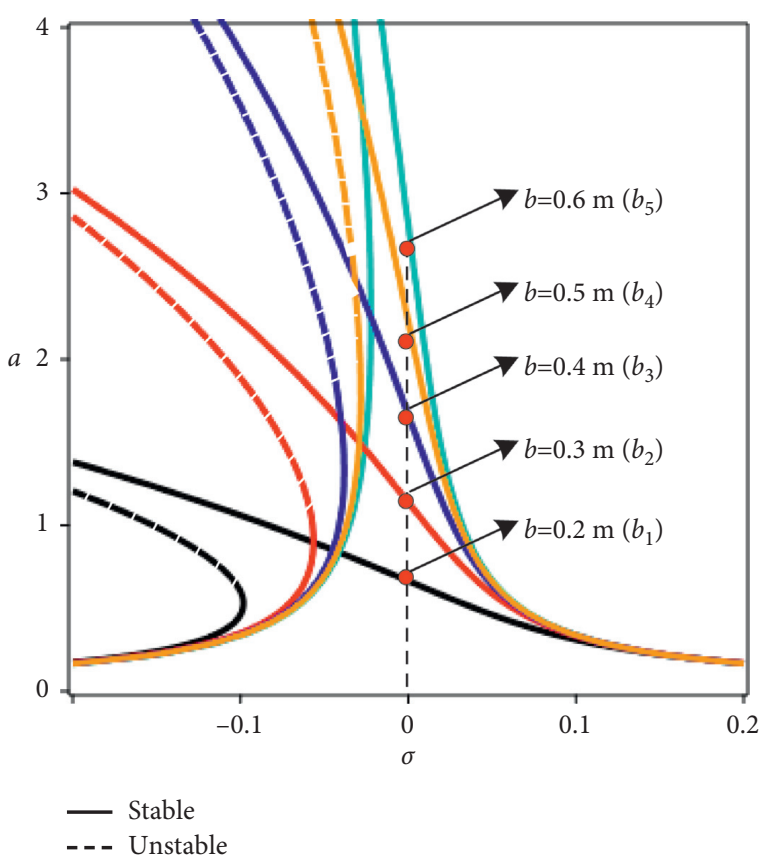

(b)

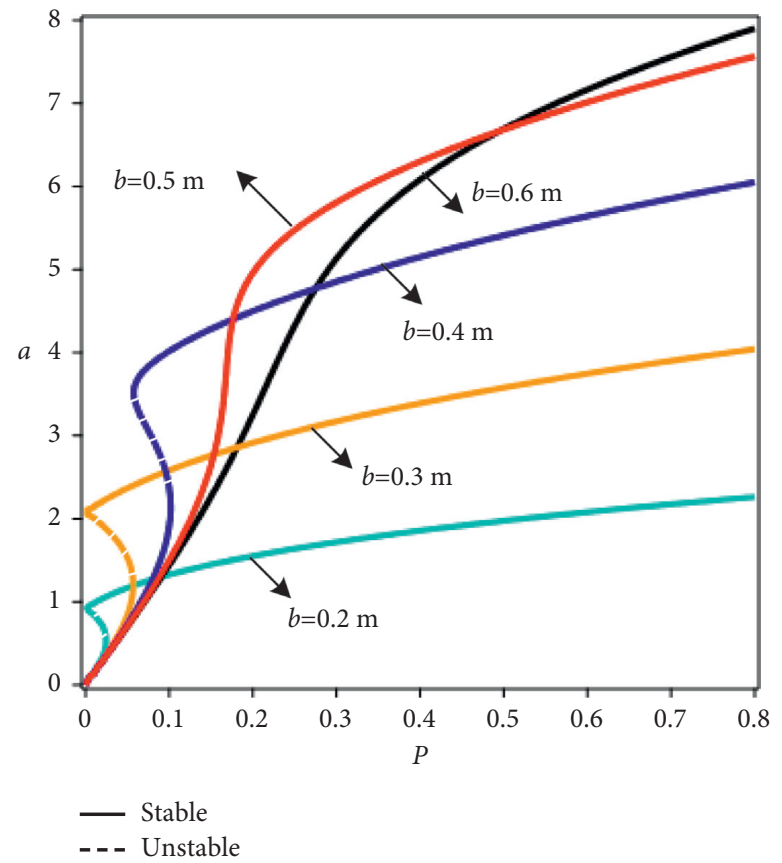

(c)

Figure 5: The amplitude-frequency response curve of primary resonance (varied b) (a) considering aerodynamic force; (b) without considering aerodynamic force; (c) with different excitation amplitudes $(\sigma=-0.1)$.

excitation conditions and initial conditions are taken, and the vibration equation (equation (18)) can be solved by using the Newton-Raphson method; then, the displacement time history curve of primary resonance can be obtained by using Runge-Kutta function in MATLAB, which is shown in Figure 6.

As shown in Figure 6, when the aerodynamic force is considered, the response amplitude is approximately
$2.13977282 \mathrm{~m}$ (it is obtained by subtracting the positive amplitude from the negative amplitude and dividing by 2 ), and the error of the theoretical solution is $1.69 \%$, in which the carrying current $i=30 \mathrm{~A}$, the direct current $I=30 \mathrm{~A}$, the spacing $b=0.5 \mathrm{~m}$, and the excitation amplitude $P=0.02916 \mathrm{~N} / \mathrm{m}$. Without considering the aerodynamic force, the response amplitude is $2.2204295 \mathrm{~m}$ (it is obtained by subtracting the positive amplitude and 


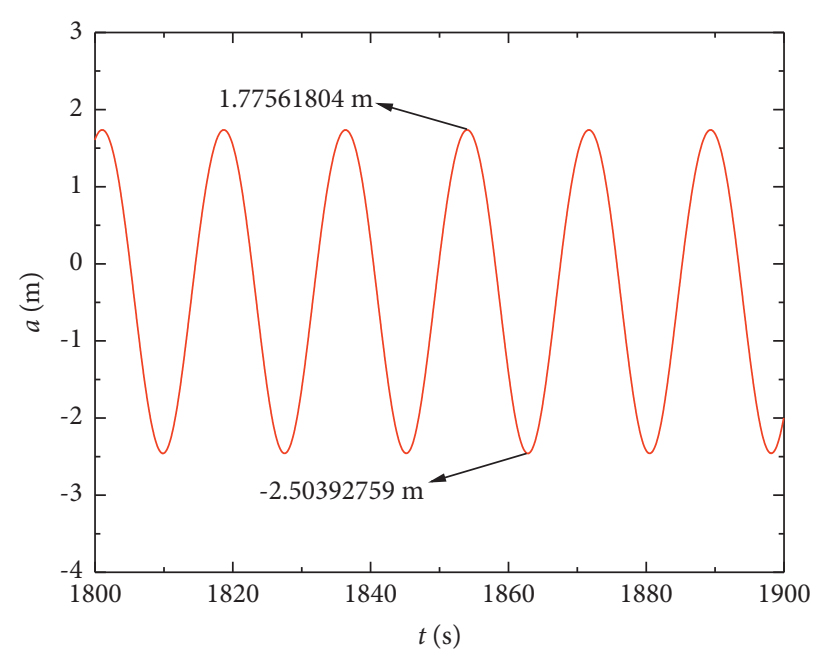

- Consider aerodynamic force

(a)

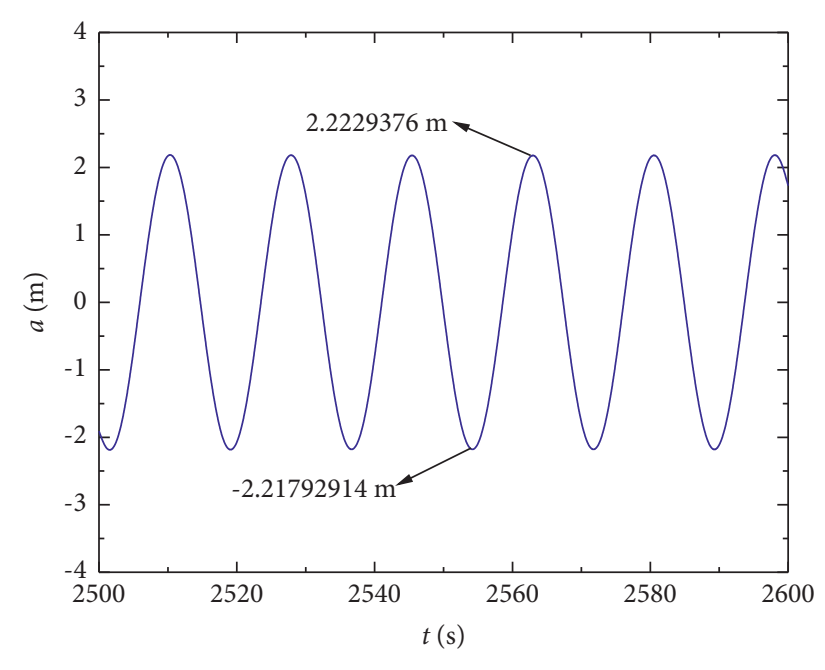

Without considering aerodynamic force

(b)

FIgURE 6: The displacement time history curve of primary resonance (a) without considering aerodynamic force and (b) considering aerodynamic force.

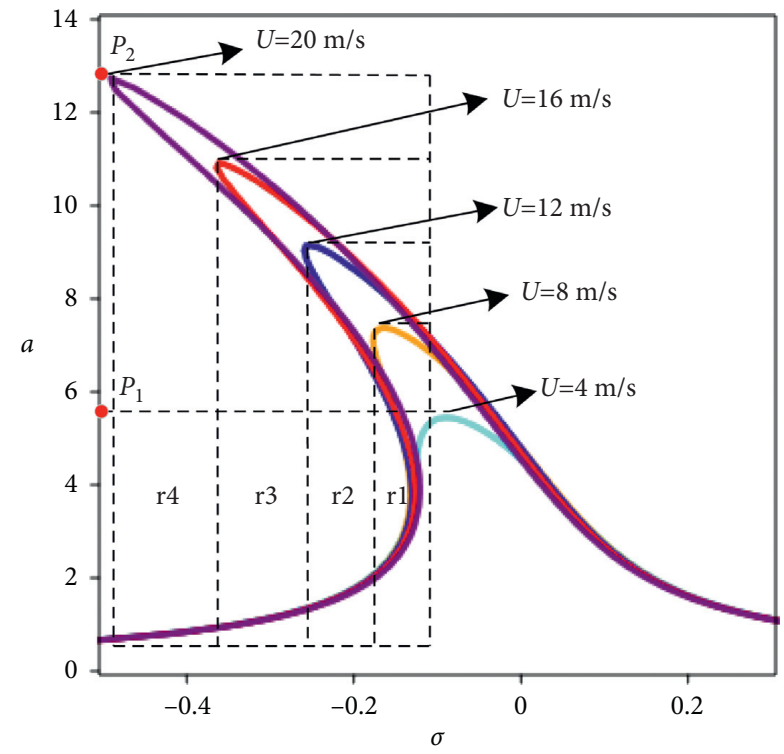

FIgURE 7: The amplitude-frequency response curve of primary resonance (varied $U$ ).

dividing it by 2), and the error with the theoretical solution is $0.589 \%$.

Combined with Figures 4 and 5, it can be seen that regardless of whether the aerodynamic force is considered, the amplitude-frequency curve presents an obvious tendency of bending to the left, which indicates that the nonlinearity of the system is strong. While the nonlinearity of the system is weakened when solving the theoretical solution, there is a certain error between the numerical solution and theoretical solution. However, the errors are all within 5\%, which indicates that the good agreements between the theoretical solutions and numerical ones.

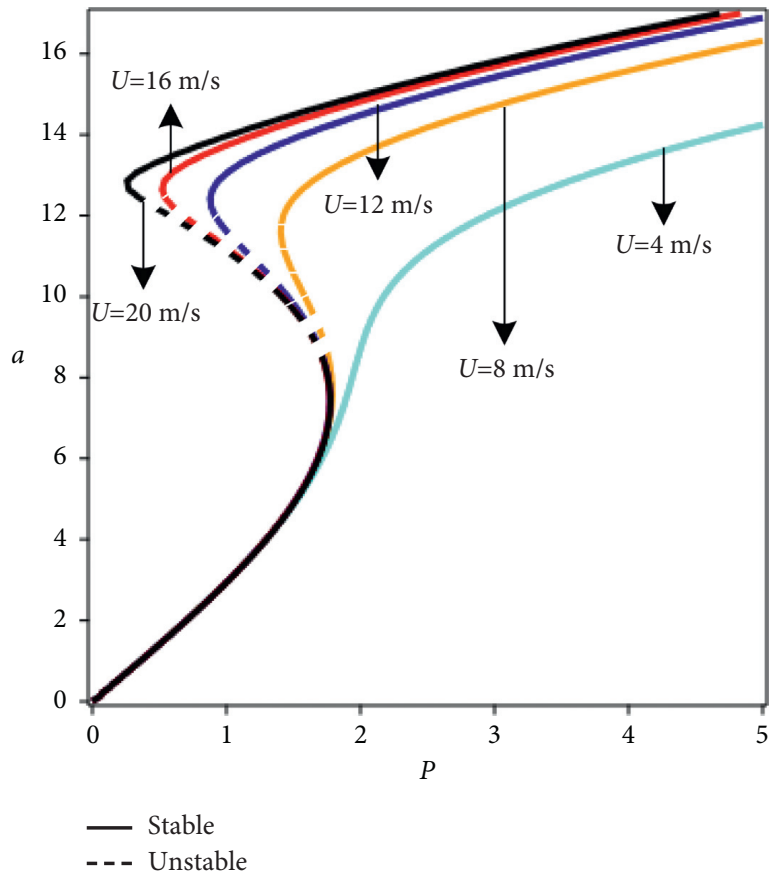

FIgURE 8: The curves of excitation amplitude $(p)$ and response amplitude ( $a$ ) (varied $U, \sigma=-0.5$ ).

Wind velocity is an important condition to induce the self-excited vibration of the iced conductors and plays a vital role in the vibration of the iced conductors. Therefore, in order to analyze the influence of wind velocity on the primary resonance of system, Figure 7 provides the frequencyamplitude curve under different wind velocities with the excitation amplitude $P=0.291678 \mathrm{~N} / \mathrm{m}$. The frequencyamplitude curves with wind velocity $U=4-20 \mathrm{~m} / \mathrm{s}$ in Figure 7 are obtained by Maple, respectively. 


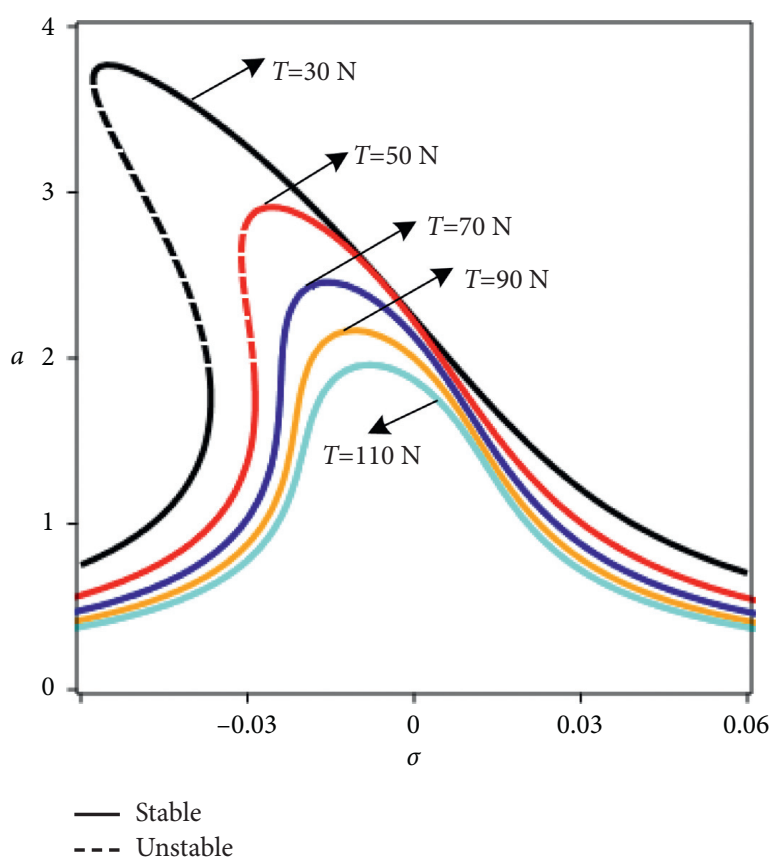

FIGURE 9: The amplitude-frequency response curve of primary resonance (varied $T$ ).

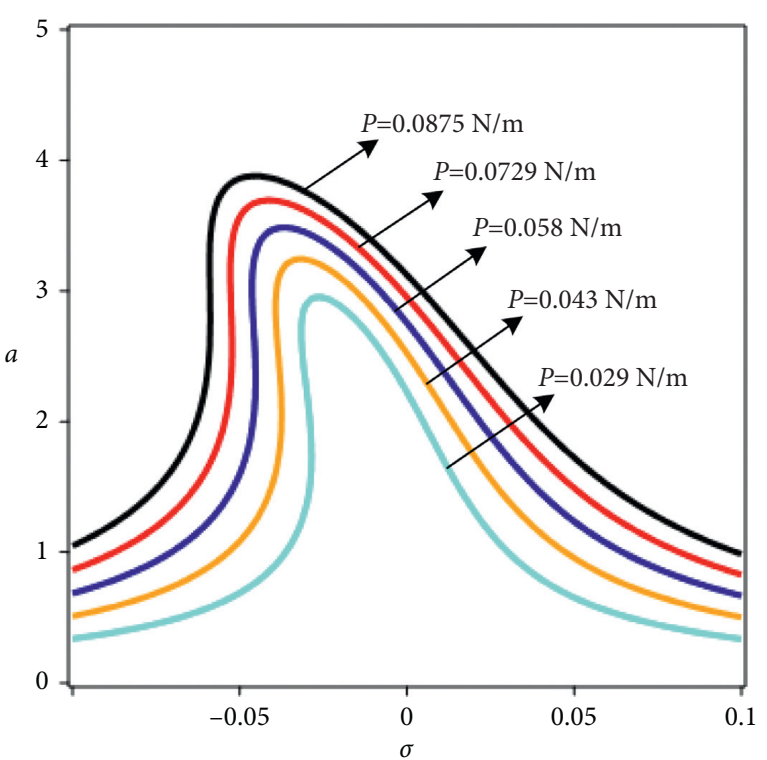

(a)

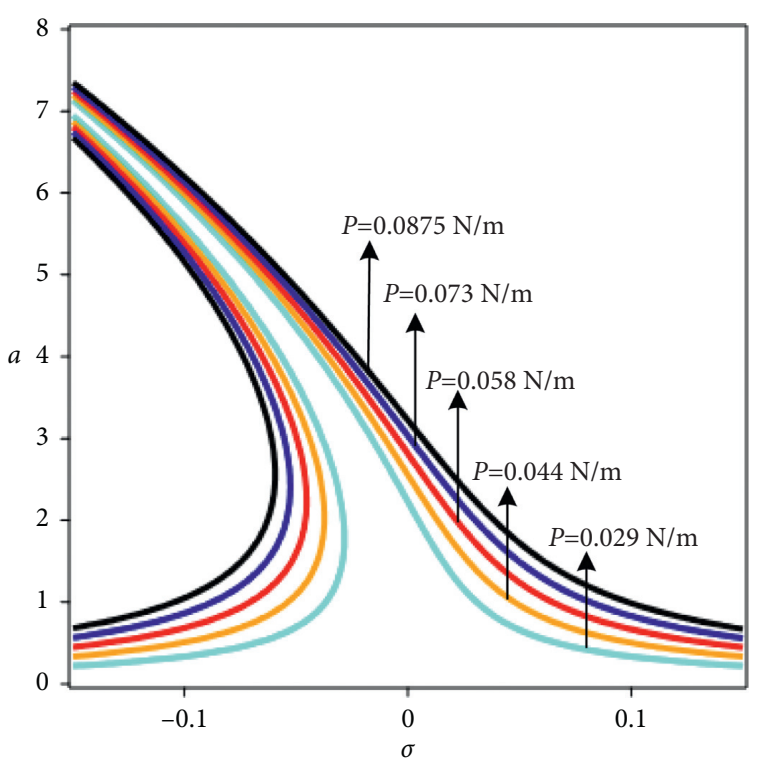

(b)

FIGURE 10: The amplitude-frequency response curve of primary resonance (varied $P$ ) (a) considering aerodynamic force; (b) without considering aerodynamic force.

In Figure 7, with the increase in wind velocity $U$, the resonance region of amplitude-frequency response curve becomes wider, and the greater the degree of curve bending to the left, it shows obvious soft spring characteristics; the peak value of the primary resonance response amplitude increases from $\mathrm{P}_{1}$ to $\mathrm{P}_{5}$, and the response amplitude is accompanied by obvious jump and multivalue phenomenon. With the increase in wind velocity, the multivalued region of amplitude-frequency curve increases from $r_{1}$ $(U=8 \mathrm{~m} / \mathrm{s})$ region to $r_{2}(U=12 \mathrm{~m} / \mathrm{s}), r_{3}(U=16 \mathrm{~m} / \mathrm{s})$, and $r_{4}$
( $U=20 \mathrm{~m} / \mathrm{s}$ ) region, and at the same time, the number of steady-state solutions increases from one to three.

In order to further analyze the influence of wind velocity on the primary resonance of the iced current-carrying conductors under the combined action of aerodynamic force-Ampere's force-forced excitation, wind velocities $U=4,8,12,16$, and $20 \mathrm{~m} / \mathrm{s}$ were, respectively, taken to draw the corresponding excitation amplitude $(p)$ and response amplitude (a) curves, in which the tuning parameter $\sigma=-0.04$, as shown in Figure 8 . 


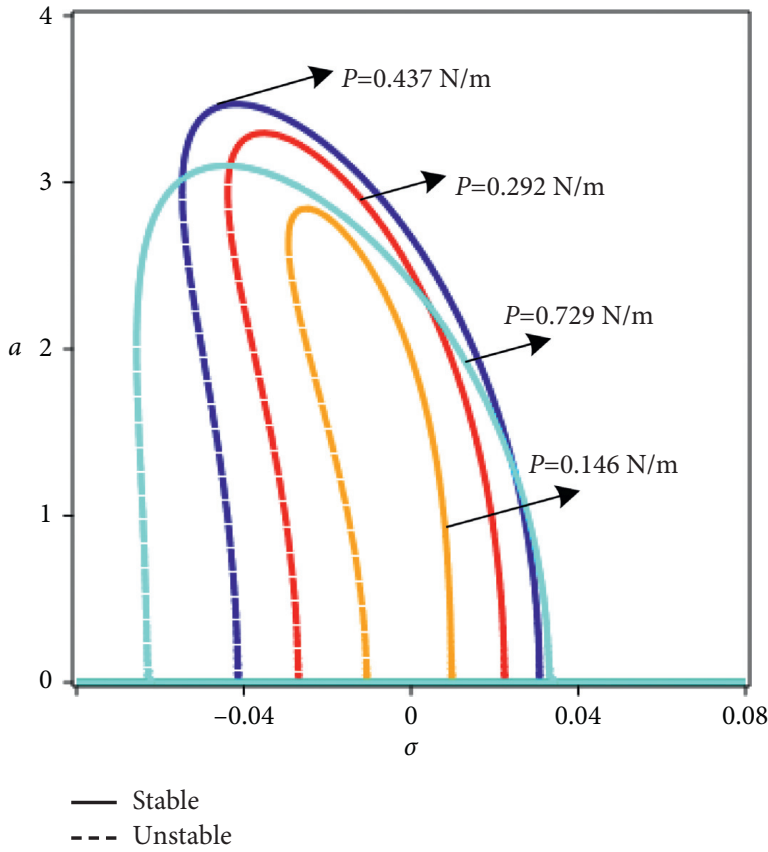

(a)

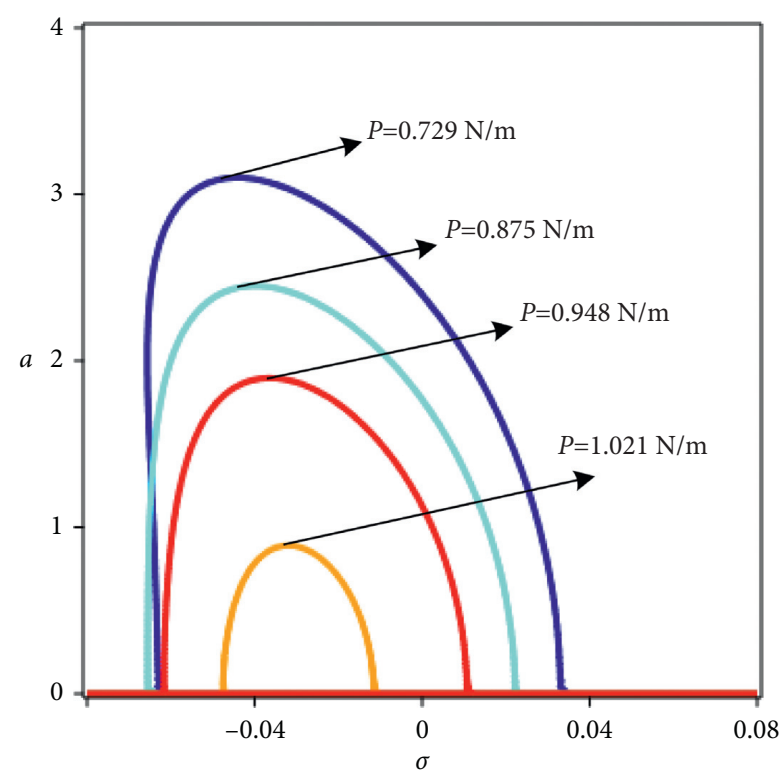

(b)

FIGURE 11: The 1/2-order subharmonic amplitude-frequency response curve (varied $P$ ) with different excitation amplitudes: (a) $0.146-0.729 \mathrm{~N} / \mathrm{m}$ and (b) $0.729-1.021 \mathrm{~N} / \mathrm{m}$.

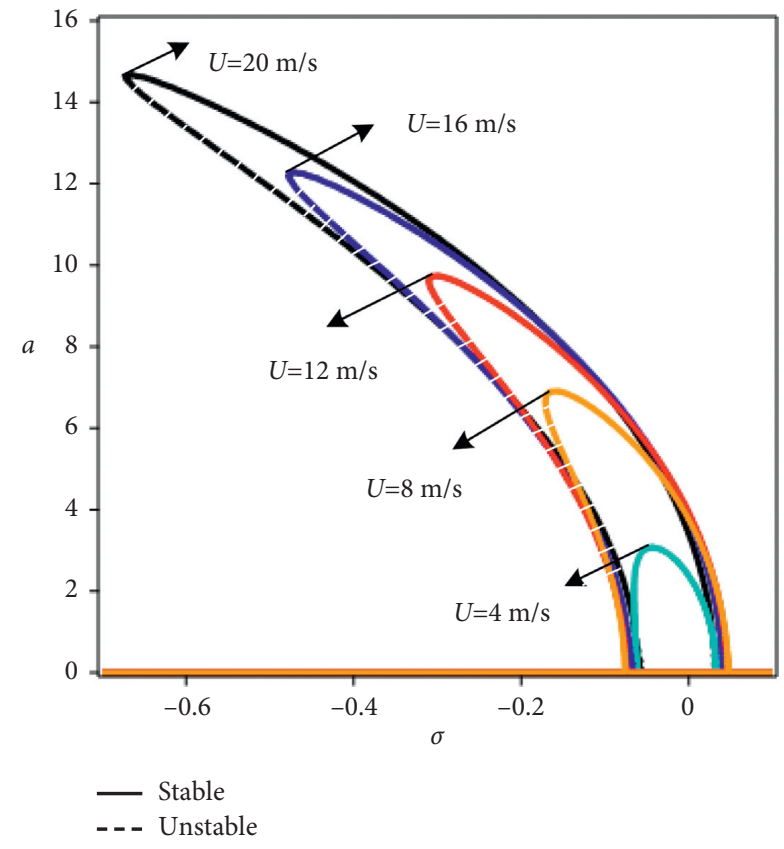

Figure 12: The 1/2-order subharmonic amplitude-frequency response curve (varied $U$ ).

It can be seen from Figure 8 that the jump and multivalue phenomenon of response amplitude are still common under different wind velocities. The $P$ - $a$ curves of the primary resonance shift to the left with the increase in wind velocity, and the multivalued region of the curve is increasing. Moreover, with the increase in wind velocity, it is

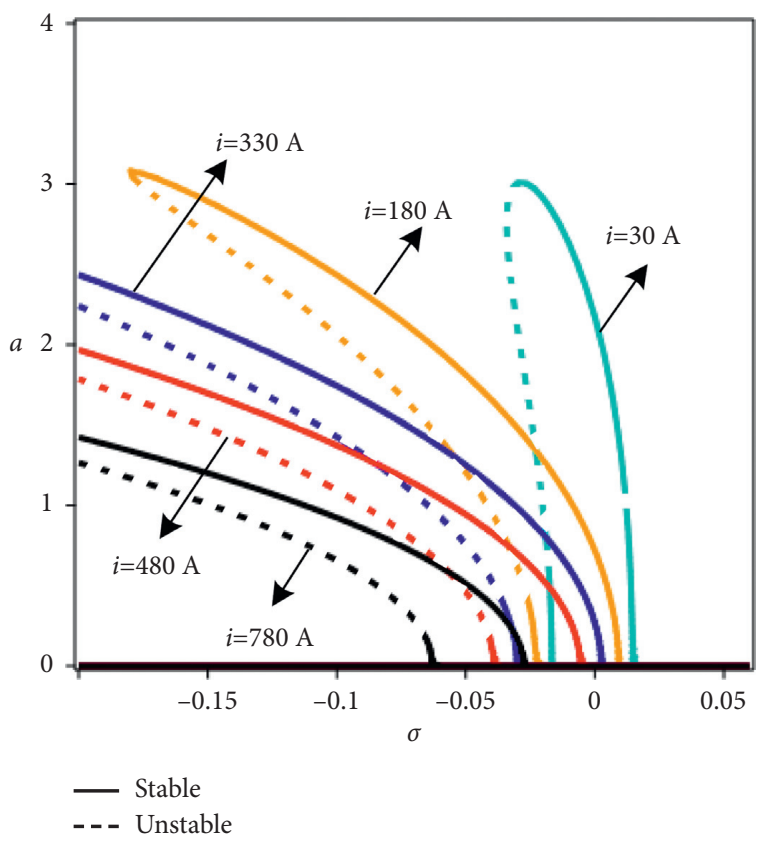

FIGURE 13: The 1/2-order subharmonic amplitude-frequency response curve (varied $i$ ).

easier to generate the jump phenomenon of response amplitude with a small excitation amplitude ( $p$ ); however, these unstable phenomena with nonlinear characteristics always occur when the excitation amplitude is limited within a certain range, and the response amplitude tends to be stable gradually with the increase in excitation amplitude. When 


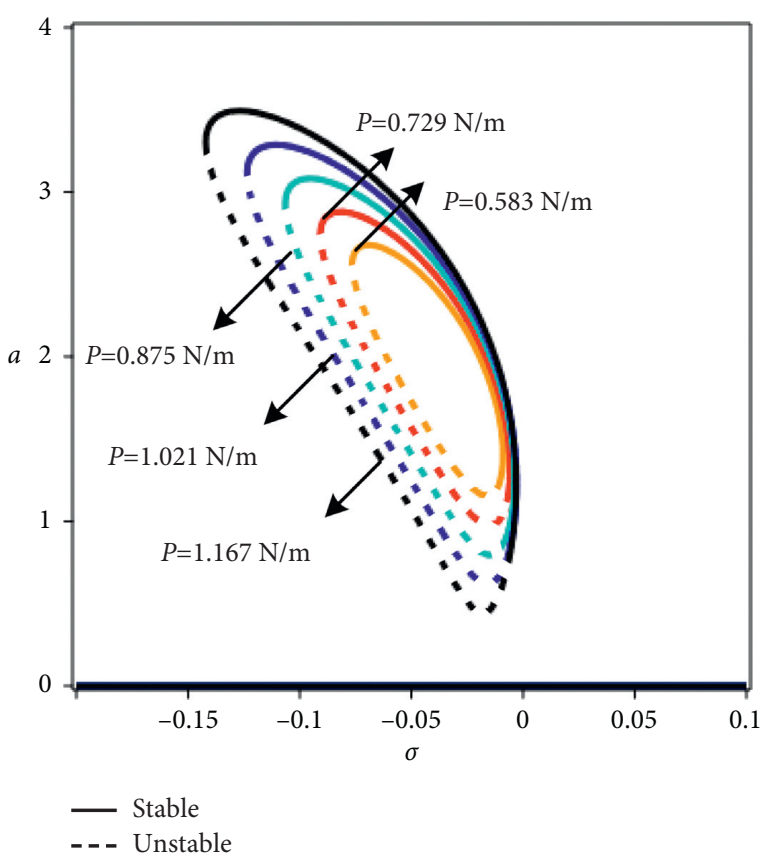

Figure 14: The 1/3-order subharmonic amplitude-frequency response curve (varied $P$ ).

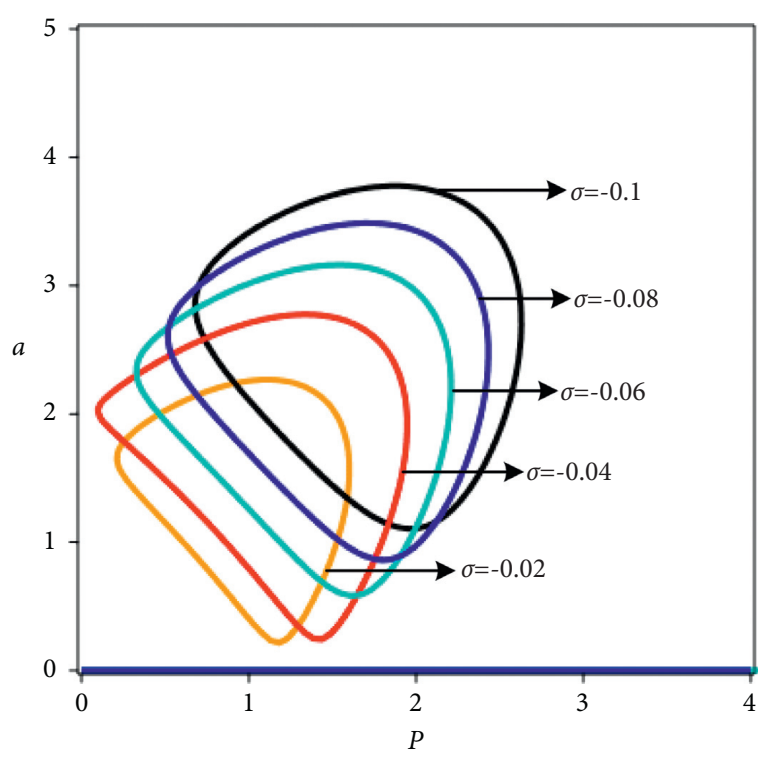

Figure 15: The curves of excitation amplitude $P$ and response amplitude $(a)$ of 1/3-order subharmonic resonance (varied $\sigma$ ).

the excitation amplitude $P>2 \mathrm{~N} / \mathrm{m}$, the response amplitude under different wind velocities increases steadily and gently with the increase in the excitation amplitude. At the same time, the greater the wind velocity, the greater the response amplitude, and when the wind velocity $U$ is in the range of 4-12 m/s, the response amplitude increases significantly, but when the wind velocity $U>12 \mathrm{~m} / \mathrm{s}$, the increase in response amplitude becomes smaller and smaller with the increase in wind velocity. These phenomena indicate that the increase in wind velocity will significantly enhance the nonlinearity of system.

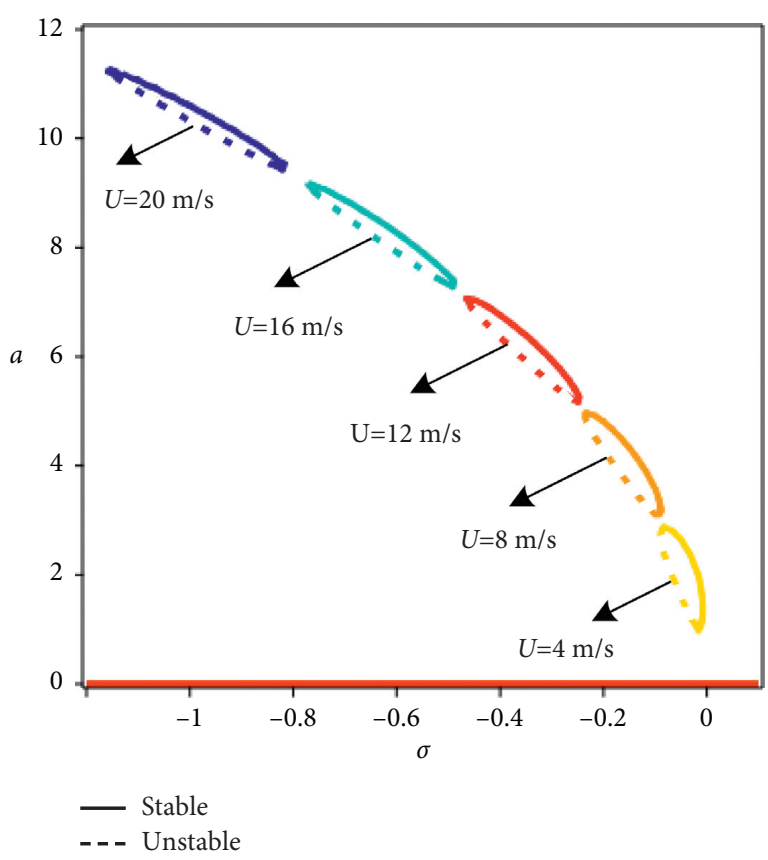

Figure 16: The 1/3-order subharmonic amplitude-frequency response curve (varied $U$ ).

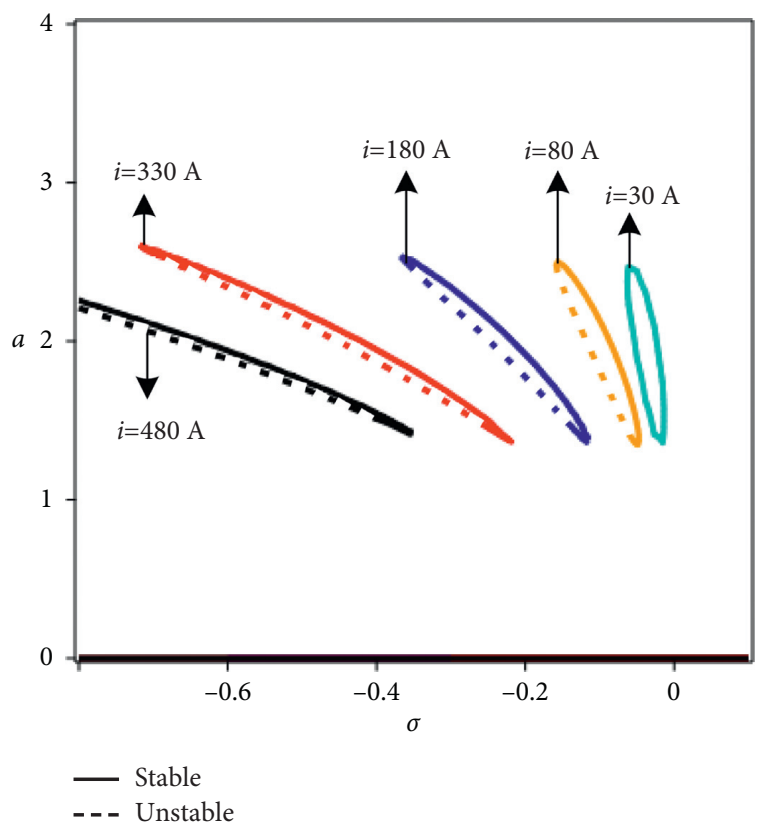

Figure 17: The 1/3-order subharmonic amplitude-frequency response curve (varied $i$ ).

According to Irvine parameter [39], the tension $T$ can affect the sag of conductor $\left(d=\left(\mathrm{mgl}^{2} / 8 T\right)\right)$ and then affect the nonlinear strength of the system. Therefore, the tension $T$ is an important parameter that cannot be ignored in the study of the nonlinear vibration characteristics of current-carrying iced conductors. When the tension $T=30,50,70,90$, and $110 \mathrm{~N}$, the amplitude-frequency response curves of the system are drawn by Maple, in which the excitation amplitude $P=0.0291678 \mathrm{~N} / \mathrm{m}$ as shown in Figure 9 . 


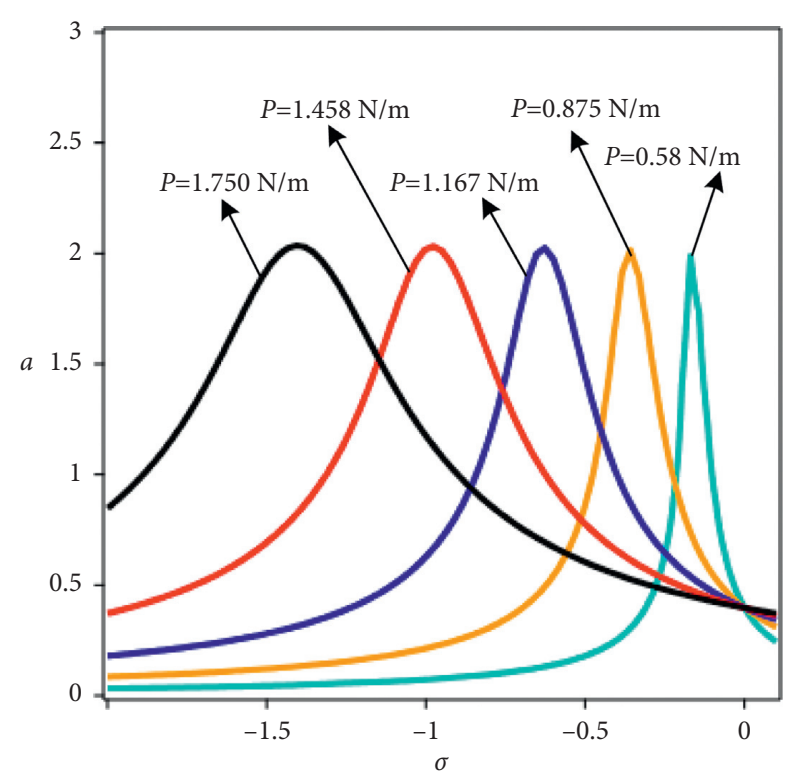

FIgURE 18: The 2-order super-harmonic amplitude-frequency response curve (varied $P$ ).

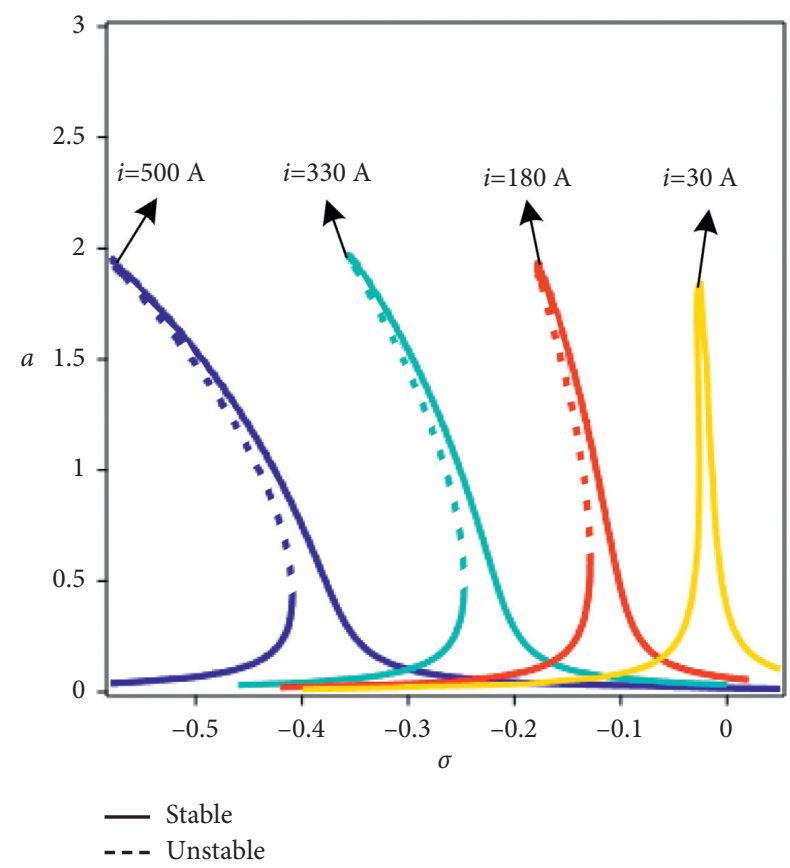

FIGURE 19: The 2-order super-harmonic amplitude-frequency response curves (varied $i$ ).

It can be obtained by analyzing Figure 9 that when the tension $T$ decreases from $50 \mathrm{~N}$ to $30 \mathrm{~N}$, the peak value of the response amplitude of the primary resonance increases significantly, the degree of the left-bending of the amplitude-frequency response curve increases, and the multivalued region of the curve also increases, and there is phenomena of jump in the amplitude, which indicates that the decrease in the tension enhances the nonlinearity of the system. However, when the tension $T$ increases from $50 \mathrm{~N}$ to $70 \mathrm{~N}$ and $90 \mathrm{~N}$ and $110 \mathrm{~N}$, respectively, the peak value of the response amplitude decreases gradually, the resonance region decreases gradually, and the curve bending degree becomes smaller and smaller. In addition, the response amplitude does not have the phenomenon of jump and multiple values, and the nonlinearity of the system weakens and becomes more stable. Therefore, in practical engineering applications, the vibration amplitude of the conductor can be reduced by increasing the tension $T$ appropriately.

As an important part of the external excitation load, the effect of the excitation amplitude on the primary resonance of the system should be analyzed in detail. Figure 10 shows the characteristics of response amplitude of primary resonance under different excitation amplitudes with the wind velocity $U=4 \mathrm{~m} / \mathrm{s}$. It is worth noting that the amplitude-frequency response curves in Figure 10(a) take into account the influence of aerodynamic force, but the amplitude-frequency response curves in Figure 10(b) do not consider the influence of aerodynamic force.

In Figure 10(a), with the increase in excitation amplitude $P$, the peak value of response amplitude increases continuously, and the resonance region becomes wider. The amplitude shows the phenomenon of jump and multiple values only within a small range of tuning parameters, and the unstable phenomenon of jumps and multiple values becomes less and less with the increase in excitation amplitude. Although the amplitude-frequency response curve has a tendency to bend to the left, the degree is relatively gentle and there is no obvious bending with the increase in the excitation amplitude. These phenomena indicate that the influence of the excitation amplitude on the vibration characteristics of the system is mainly reflected in the response amplitude. In combination with Figures 10(a) and 10(b), it can be found that the peak value of the response amplitude of the primary resonance is also increasing without considering the aerodynamic force. However, compared with the situation of considering the aerodynamic force, the bending degree of amplitude-frequency curve without considering aerodynamic force is greater, which indicates that the nonlinearity of system without considering aerodynamic force is much stronger. And after considering the aerodynamic force, the bending degree of amplitude-frequency curve is noticeably reduced, and this indicates that aerodynamic force weakens the nonlinearity of the forced system to a certain extent.

6.1.1. Stability Analysis of Primary Resonance. Equation (32) is written as

$$
4 \kappa\left(\sigma+\frac{3 \kappa \vartheta_{m}}{2 \omega_{0}}\right)^{2}+\kappa\left(\vartheta_{k}+3 \kappa \vartheta_{v} \omega_{0}^{2}\right)^{2}=H
$$

where $\kappa=a^{2} / 4, H=P^{2} /\left(4 \omega_{0}^{2}\right)$.

By linearizing equations (31a) and (31b) at $(a, \varphi)$, we can get 


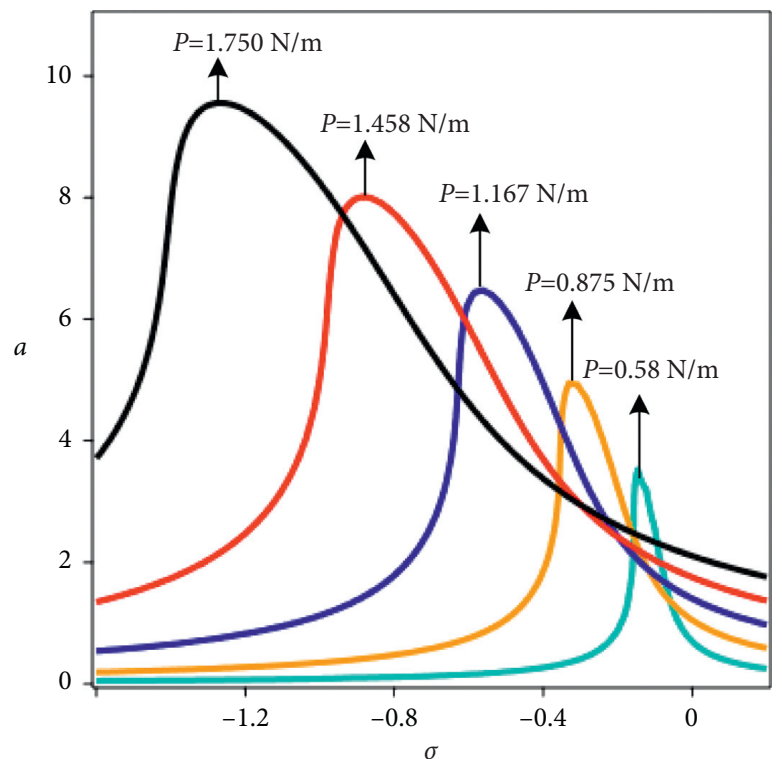

- Stable

-.- Unstable

(a)

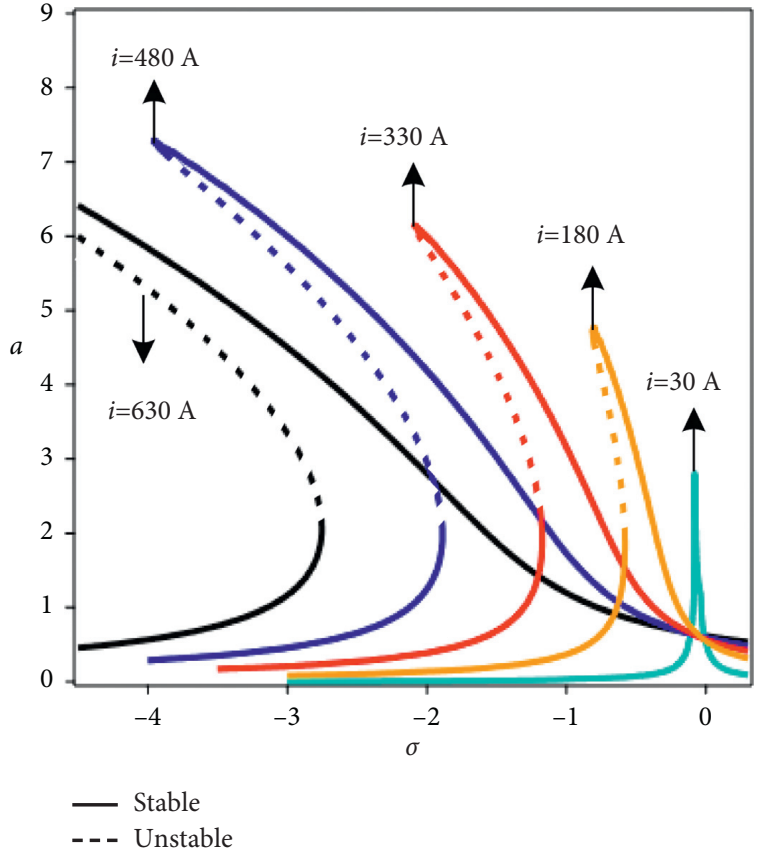

(b)

FIGURE 20: The 3-order super-harmonic amplitude-frequency response curve (a) with different excitation amplitudes; (b) different currentcarrying.

TABLE 1: The amplitude of considering aerodynamic force and not considering aerodynamic force (varied $i, \sigma=0$ ).

\begin{tabular}{lcccc}
\hline$i_{55}$ & $i_{44}$ & $i_{33}$ & $i_{22}$ & $i_{11}$ \\
$2.1770 \mathrm{~m}$ & $1.2171 \mathrm{~m}$ & $0.9975 \mathrm{~m}$ & $0.8823 \mathrm{~m}$ & $0.7527 \mathrm{~m}$ \\
\hline$i_{5}$ & $i_{4}$ & $i_{3}$ & $i_{2}$ & $i_{1}$ \\
$2.2336 \mathrm{~m}$ & $1.2381 \mathrm{~m}$ & $1.0125 \mathrm{~m}$ & $0.8939 \mathrm{~m}$ & $0.7606 \mathrm{~m}$ \\
\hline
\end{tabular}

$$
\left\{\begin{array}{l}
D_{1} \Delta a=\frac{\Delta a \cdot \vartheta_{k}}{2}+\frac{9 \vartheta_{v} \omega_{0}^{2} a^{2} \cdot \Delta a}{8}-\frac{P \cos \varphi \cdot \Delta \varphi}{2 \omega_{0}} \\
D_{1} \varphi=\frac{6 \vartheta_{m} a \cdot \Delta a}{8 \omega_{0}}+\frac{P \cos \varphi \cdot \Delta a}{2 \omega_{0} a^{2}}+\frac{P \sin \varphi \cdot \Delta a}{2 \omega_{0} a}
\end{array}\right.
$$

With equation (31) to eliminate $\cos \phi$ and $\sin \phi$ in the above equation, the characteristic equation is obtained:

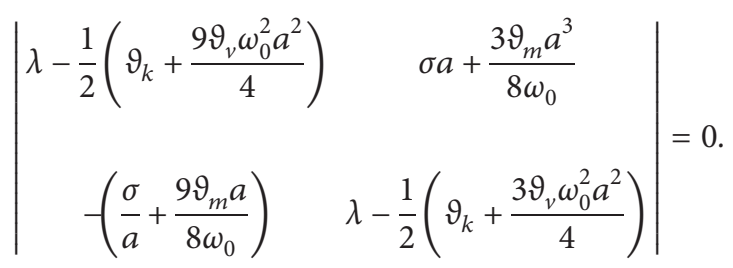

Expanding the determinant and simplifying it, we can get

$$
\lambda^{2}-v_{n} \lambda+Q_{n}=0
$$

where
TABle 2: The amplitude of considering aerodynamic force and not considering aerodynamic force (varied $b, \sigma=0$ ).

\begin{tabular}{lcccc}
\hline$b_{11}$ & $b_{22}$ & $b_{33}$ & $b_{44}$ & $b_{55}$ \\
$0.6577 \mathrm{~m}$ & $1.1204 \mathrm{~m}$ & $1.6386 \mathrm{~m}$ & $2.1770 \mathrm{~m}$ & $2.5784 \mathrm{~m}$ \\
\hline$b_{1}$ & $b_{2}$ & $b_{3}$ & $b_{4}$ & $b_{5}$ \\
$0.6642 \mathrm{~m}$ & $1.1391 \mathrm{~m}$ & $1.6667 \mathrm{~m}$ & $2.2315 \mathrm{~m}$ & $2.8186 \mathrm{~m}$ \\
\hline
\end{tabular}

$$
\begin{aligned}
& \nu_{n}=\vartheta_{k}+6 \kappa \vartheta_{v} \omega_{0}^{2} \\
& Q_{n}=\sigma^{2}+\frac{1}{4} \vartheta_{k}^{2}+3 \vartheta_{k} \vartheta_{v} \omega_{0}^{2} \kappa+\frac{27}{4} \vartheta_{v}^{2} \omega_{0}^{4} \kappa^{2}+\frac{6 \vartheta_{m} \sigma \kappa}{\omega_{0}}+\frac{27 \vartheta_{m}^{2} \kappa^{2}}{4 \omega_{0}^{2}}
\end{aligned}
$$

Solve equation (50) to obtain

$$
\begin{aligned}
\lambda_{1,2} & =\frac{1}{2}\left(v_{n} \pm \sqrt{v_{n}^{2}-4 Q_{n}}\right) \\
& =\frac{1}{2}\left(\vartheta_{k}+6 \kappa \vartheta_{v} \omega_{0}^{2} \pm \sqrt{9 \vartheta_{v}^{2} \omega_{0}^{4} \kappa^{2}-4 \sigma^{2}-\frac{24 \vartheta_{m} \sigma \kappa}{\omega_{0}}-\frac{27 \vartheta_{m}^{2} \kappa^{2}}{\omega_{0}^{2}}}\right) .
\end{aligned}
$$

The condition of the above equation has negative real parts as

$$
\begin{gathered}
v_{n}<0 ; \\
Q_{n}>0 .
\end{gathered}
$$

Setting $v n=0$ and $Q n=0$, Figure 21 is obtained. As shown in Figure 21, the nonshadowed region formed by the 
horizontal straightness $\kappa=0.451379$ and the ellipse $Q n=0$ meets the condition of steady-state motion stability. Two oblique lines are obtained by the critical condition $\kappa=-25.228 \sigma$ and $\kappa=-113.878 \sigma$ for whether $\lambda_{1,2}$ is a complex root or not. At the same time, the point within the ellipse is the saddle point, and the point between the ellipse and the sector is the node.

By analyzing Figure 21, it can be found that the behavior of amplitude-frequency response curves can be classified as follows: when the tuning parameter $\sigma>0$, no matter what the excitation amplitude is, equation (47) has only one steadystate solution and it is unstable. When the tuning parameter $\sigma<0$, with the change of its own absolute value and excitation amplitude, it can be divided into the following situations.

$0<H<0.000723$ : when the tuning parameter $\sigma$ is very small or relatively large, equation (47) has only one steady-state solution and it is unstable; when the tuning parameter $\sigma$ is slightly small, equation (47) has three steady-state solutions, but only the solution with the largest amplitude is asymptotically stable; $0.000723<H$ : when the tuning parameter $\sigma$ is small, equation (47) has only one steady-state solution and it is asymptotically stable.When the tuning parameter $\sigma$ is large, there is only one unstable steady-state solution to equation (47); to sum up, only when the tuning parameter $\sigma$ is small, and the response amplitude of the system will not show the phenomena of jump but will keep an asymptotically stable synchronous vibration. If the tuning parameter $\sigma$ value is a little larger, the response amplitude will show the out-of-step phenomenon, as shown in Figure 22.

\subsection{Harmonic Resonance}

6.2.1. 1/2-Order Subharmonic Response. In order to analyze the influence of excitation amplitude on the 1/2-order subharmonic response of the iced current-carrying conductors under the combined action of aerodynamic force, Ampere's force, and forced excitation, the amplitude-frequency response curve of equation (43) is drawn by Maple in Figure 11.

As shown in Figure 11(a), when the excitation amplitude $P$ is in the range of $0.146 \mathrm{~N} / \mathrm{m}$ to $0.437 \mathrm{~N} / \mathrm{m}$, the peak value of response amplitude increases with the increase in excitation amplitude, and the response amplitude shows the characteristics of multivalued solution. Moreover, the peak value of response amplitude keeps moving towards the negative value of tuning parameter $\sigma$, and the resonance region also keeps increasing. The left-bending degree of the curve increases with the increase in the excitation amplitude, showing the characteristics of soft spring. However, when the excitation amplitude changes from $P=0.437 \mathrm{~N} / \mathrm{m}$ rise to $0.729 \mathrm{~N} / \mathrm{m}$, the peak value of the response amplitude decreases. In Figure 11(b), when the excitation amplitude $P$ is in the range of $0.729 \mathrm{~N} / \mathrm{m}$ to $1.021 \mathrm{~N} / \mathrm{m}$, the peak value of the response amplitude decreases with the increase in the excitation amplitude, and the resonance region shifts towards the negative direction of the tuning parameter $\sigma$. However, the softening phenomenon of the system gradually decreases with the increase in the excitation amplitude.

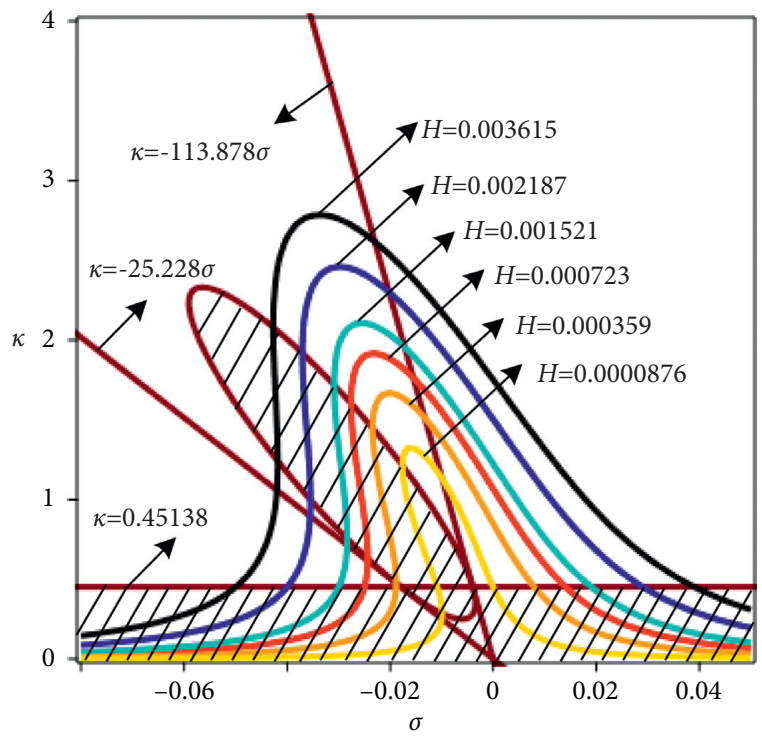

FIgURE 21: The amplitude-frequency response curve under different excitation amplitudes.

On the whole, the response amplitude of the $1 / 2$-order subharmonic resonance increases first and then decreases, and the jump and multivalue phenomenon of the response amplitude become less and less and even disappear. The softening phenomenon of the system increases first and then decreases gradually with the increase in the excitation amplitude. The resonance region will shift when the excitation amplitude increases to a certain range.

The 1/2-order subharmonic amplitude-frequency response curve varying with wind velocity is drawn by Maple in Figure 12, in which the excitation amplitude $P=0.729 \mathrm{~N} /$ $\mathrm{m}$ and the wind velocity $U$ started at $4 \mathrm{~m} / \mathrm{s}$ and increased by $4 \mathrm{~m} / \mathrm{s}$ each time until $U=20 \mathrm{~m} / \mathrm{s}$.

As shown in Figure 12, with the increase in wind velocity $U$, the peak value of response amplitude increases, the degree of the amplitude-frequency curve bending to the left is getting bigger and bigger, and the resonance peak offsets towards the negative direction of horizontal coordinate axis $\sigma$, showing soft spring characteristics, and the response amplitude has obvious the multivalue and jump phenomenon. By comparing Figures 11 and 12, it can be seen that the influence of excitation amplitude on the system is mainly reflected in the value of response amplitude, while the influence of wind velocity on the system is not only reflected in the value of amplitude but also has a direct influence on the nonlinearity of the system.

Figure 13 discusses the characteristics of response amplitude on the 1/2-order subharmonic resonance under different current-carrying, in which the excitation amplitude $P=0.19445 \mathrm{~N} / \mathrm{m}$.

As shown in Figure 13, with the increase in currentcarrying $i$, the resonance region of the amplitude-frequency response curve shows obvious offset, and the peak value of the response amplitude also deviates towards the negative direction of horizontal coordinate axis $\sigma$, and the degree of left-bending becomes more and more obvious, which 


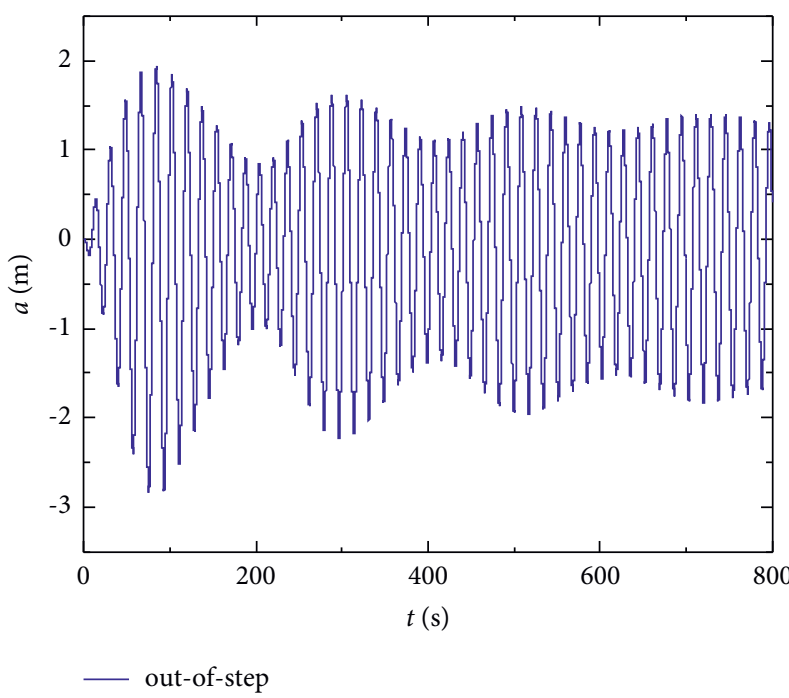

(a)

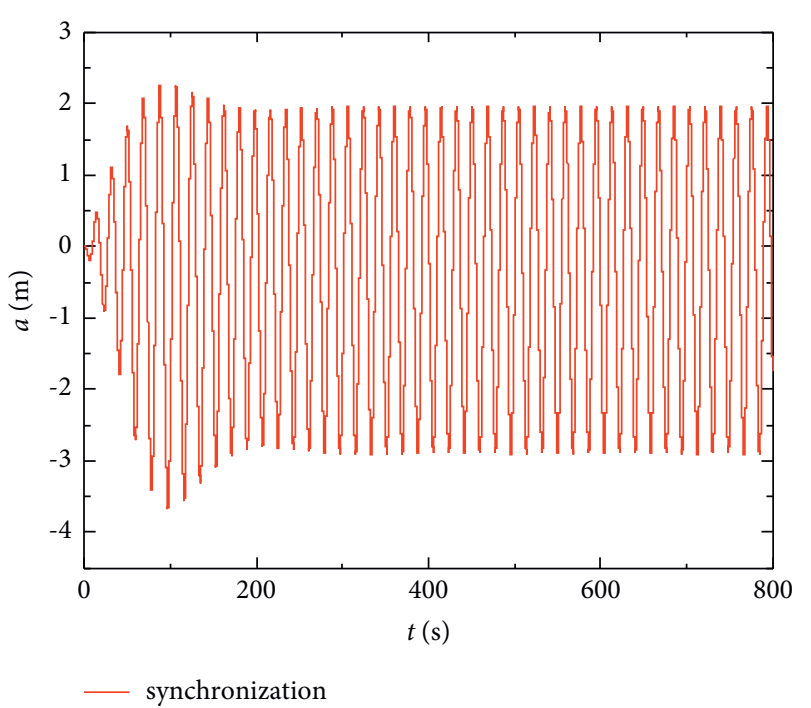

(b)

FIgURE 22: The displacement time history curve of primary resonance. (a) Synchronization $(\sigma=-0.01)$. (b) Out-of-step ( $\sigma=0.15$ ).

indicates that the nonlinearity of the system is stronger and stronger. At the same time, with the increase in currentcarrying $i$, the response amplitude is accompanied by the complex nonlinear dynamic behavior of multiple value and jump. However, the peak value of the response amplitude does not increase with the increase in current-carrying $i$ but basically remains unchanged. In addition, when the tuning parameter $\sigma>0$, the response amplitude of 1/2-order subharmonic resonance will decrease with the increase in current-carrying $i$. When the tuning parameter $\sigma<0$, the response amplitude of the $1 / 2$-order subharmonic resonance will increase with the increase in the current-carrying $i$.

6.2.2. 1/3-Order Subharmonic Response. In order to analyze the influence of excitation amplitude on the 1/3-order subharmonic response of the iced current-carrying conductors under the combined action of aerodynamic forceAmpere's force-forced excitation, when the system shows 1/ 3 -order subharmonic resonance, the amplitude-frequency curves are obtained by substituting the excitation amplitude $P=1.868-22.416 \mathrm{~N} / \mathrm{m}$ and other parameters into equation (44).

As shown in Figure 14, the shape of the amplitudefrequency curve resembles the shape of a closed ellipse. With the increase in excitation amplitude $P$, the peak value of the response amplitude increases, and the resonance region of $1 /$ 3 -order subharmonic resonance curve continues to expand. The degree of left-bending of curve becomes greater, and the peak value of the response amplitude also shifts to the negative direction of horizontal coordinate axis $\sigma$ as the excitation amplitude increases, showing the nonlinear characteristics of softening behavior. It can be clearly observed that in the vast majority of cases, the response amplitude of the system exists in the form of multiple solutions, and there are jump phenomena. At the same time, in combination with equation (44), it can be found that there is a case where the value of amplitude is zero on 1/3-order of the subharmonic resonance.

In order to further analyze the effect of excitation amplitude on 1/3-order subharmonic resonance, Figure 15 compares the excitation amplitude $(p)$-response amplitude (a) curves of the 1/3-order subharmonic resonance under different tuning parameters.

As shown in Figure 15, as the absolute value of tuning parameter $\sigma$ increases from -0.02 to -0.1 , the excitation amplitude is required to excite $1 / 3$-order subharmonic resonance and becomes larger and larger. Similarly, in most cases, the response amplitude exists in the form of multiple solutions, and there is the phenomenon of jump. When the tuning parameter is equal to 0 , the response amplitude has only zero solution. These conclusions are consistent with those in Figure 14.

Figure 16 shows the amplitude-frequency response curves of $1 / 3$-order subharmonic resonance under different wind velocities, in which the excitation amplitude $P=0.729 \mathrm{~N} / \mathrm{m}$. As the wind velocity increases from $4 \mathrm{~m} / \mathrm{s}$ to $20 \mathrm{~m} / \mathrm{s}$, the peak value of the response amplitude increases and shifts to the negative direction of horizontal coordinate axis $\sigma$. The amplitude-frequency curve gradually changes from a smooth ellipse to a slim ellipse and the degree of leftbending becomes bigger, which indicates that the nonlinearity of the system is stronger and stronger.

The influence of the change of the current-carrying $i$ on the nonlinear vibration characteristics of the system is one of the key research parameters in this paper. Therefore, the current-carrying $i$ cannot be ignored when studying the influence of various parameters on the 1/3-order subharmonic resonance characteristics.

Figure 17 shows the amplitude-frequency response curves of the 1/3-order subharmonic resonance with the change of the current carrying $i$, in which the excitation amplitude 
$P=0.4375 \mathrm{~N} / \mathrm{m}$. As shown in Figure 17, the amplitude-frequency response curves of the 1/3-order subharmonic resonance that changes with the current-carrying $i$ are a flat ellipse. With the increase in the current-carrying $i$, the resonance peak shifts to the negative direction of the horizontal axis $\sigma$. However, unlike the primary resonance and the 1/2-order subharmonic resonance in which the resonance peak value will increase with the increase in the current-carrying $i$, the resonance peak value of the 1/3-order subharmonic resonance does not change with the increase in the current-carrying $i$. With the increase in current-carrying $i$, the amplitude-frequency response curve becomes more and more obvious to the left, and the response amplitude has the phenomenon of multivalue and jump, indicating that the increase in current-carrying $i$ will increase the nonlinearity of the system.

6.2.3. 2-Order Super-Harmonic Response. When the current-carrying iced conductors show 2-order super-harmonic resonance, the corresponding parameters are substituted into equation (45) to obtain the amplitude-frequency response curves of the 2-order super-harmonics under different excitation amplitudes, in which the wind velocity $U=4 \mathrm{~m} / \mathrm{s}$, as shown in Figure 18 .

In Figure 18, as the excitation amplitude $P$ increases from $0.583 \mathrm{~N} / \mathrm{m}$ to $1.750 \mathrm{~N} / \mathrm{m}$, the resonance region of the amplitudefrequency curve increases and shifts towards the negative direction of the horizontal coordinate $\sigma$. Although the peak value of the response amplitude is also shifted along with the resonance region, the peak size of the response amplitude does not change. At the same time, with the increase in excitation amplitude, the response amplitude does not have the phenomenon of multivalue and jump.

Figure 19 shows the amplitude-frequency response curves of the 2-order super-harmonic resonance under different carrying current $i$, in which the excitation amplitude $P=0.1944 \mathrm{~N} / \mathrm{m}$. As shown in Figure 19, by comparing the characteristics of response amplitude of the 2-order superharmonic resonance, the primary resonance, and the subharmonic resonance under different carrying-current $i$, it can be obtained that the amplitude-frequency response curve of the 2order super-harmonics resonance has narrower resonance region and more obvious offset, and only when the value of current-carrying $i$ is larger, the curve will show obvious bending. The multivalue region of the response amplitude is less. With the increase in current-carrying $i$, the resonance peak of the 2order super-harmonic resonance does not show the phenomenon that the resonance peak of the $1 / 2$ subharmonic resonance increases significantly. On the contrary, its resonance peak value basically does not change. When the tuning parameter $\sigma>0$, the response amplitude of the 2-order superharmonic resonance will decrease with the increase in the carrying current $i$. However, when the tuning parameter $\sigma<0$, the response amplitude of the 2-order super-harmonic resonance will increase with the increase in the current-carrying $i$.

6.2.4. 3-Order Super-Harmonic Response. In order to analyze the influence of wind velocity, excitation amplitude, and current-carrying on the 3-order super-harmonic resonance of iced current-carrying conductors, the frequency-amplitude curve of equation (46) is drawn by Maple as shown in Figures 20(a) and 20(b).

As shown in Figure 20(a), the influence of excitation amplitude $P$ on the response amplitude of the 3-order superharmonic resonance is similar to that the phenomenon of the 2-order super-harmonic resonance. The difference is that the peak value of response amplitude of the 3-order superharmonic resonance increases obviously as the excitation amplitude $P$ increases from $0.583 \mathrm{~N} / \mathrm{m}$ to $1.458 \mathrm{~N} / \mathrm{m}$. And with the increase in excitation amplitude $P$, there is a clear shift of the resonance region. At the same time, the amplitude-frequency curve shows a certain degree of bending, and the resonance peak also shifts to the negative direction of the horizontal axis $\sigma$, showing softening characteristics. Compared with the 2-order super-harmonic resonance, under the same initial conditions and excitation amplitude, the response amplitude of the 3-order super-harmonic resonance is significantly larger, and the resonance region is also wider.

As shown in Figure 20(b), with the increase in currentcarrying $i$ (excitation amplitudes $P=0.4375 \mathrm{~N} / \mathrm{m}$ ), the resonance region expands and shows obvious shift, and the resonance peak also increases significantly; when the current-carrying $i$ is small, the response amplitude does not exist the multivalued region; when the carrying current $i$ increases to $130 \mathrm{~A}$, the response amplitude shows obvious the multivalue and jump phenomenon, and the curve bends to the left, showing the characteristics of softening behavior; these phenomena show that the increase in current-carrying $i$ also significantly increases the nonlinearity of the system.

\section{Conclusion}

A new current-carrying iced conductor system under the combined action of Ampere's forces, forced excitation, and aerodynamic forces has been formulated, in which forced excitation was introduced to study the influence of dynamic wind on the nonlinear vibration characteristics of currentcarrying iced conductors. Thus, the theoretical model is improved, and a new self-excited Ampere's force-forced system has been proposed. The theoretical solution is solved by the multiscale method and compared with the numerical solution. Finally, the nonlinear forced primary resonance and harmonic resonance of the current-carrying iced conductors under the combined action of aerodynamic force, Ampere's force, and forced excitation are systematically studied through the analysis of specific examples. The following conclusions can be listed:

(1) In the case of primary resonance response, regardless of whether the aerodynamic force is considered, the response amplitude of the system will decrease with the increase in the current-carrying $i$, and the degree of left-bending of the amplitude-frequency curve becomes bigger, showing soft spring characteristics, and the response amplitude is accompanied by jump and multivalue phenomena; on the contrary, the response amplitude of the system will increase with 
the increase in the spacing $b$; the value of amplitude is larger without consideration of aerodynamic force than that with consideration of aerodynamic force, and the degree of crook of amplitude-frequency curve is larger, which indicates that the nonlinearity of the system is weaker with consideration of aerodynamic force than that without consideration of aerodynamic force. When the value of the tuning parameter $\sigma$ is small, the system will have stable synchronous vibration. When the value of the tuning parameter $\sigma$ is a little larger, the out-of-step phenomenon will appear.

(2) The excitation amplitude needed to excite the harmonic resonance is larger than the primary resonance. And with the increase in the excitation amplitude, the response amplitude gradually shows the multivalue and jump phenomenon. However, it is different from other harmonic resonance responses that the peak value of the response amplitude of the 2-order super-harmonic resonance response does not increase or decrease but basically stays the same. The resonance region expands and shifts to the negative direction of the horizontal coordinate $\sigma$ with the increase in the excitation amplitude. When the system shows harmonic resonance, the increase in current carrier $i$ will enhance the nonlinearity of the system, and when the current-carrying $i$ increases to a certain value, the response amplitude will also show obvious multivalue.

(3) By comparing the two kinds of harmonic resonance under the same excitation conditions and the initial conditions, it can be found that the frequency range of super-harmonic resonance and subharmonic resonance excited by the system is different. The response amplitude of the super-harmonic resonance increases more, but the excitation conditions for reaching the peak of the super-harmonic resonance are harsher than those of harmonic resonance.

(4) The increase in wind velocity $U$ has roughly the same influence on primary resonance and harmonic resonance: the peak value of the response amplitude is getting larger and larger, and the resonance peak is shifted to the negative direction of the horizontal coordinate axis $\sigma$, showing soft spring characteristics; the multivalue region of the response amplitude is also increasing, and the degree of curve bending to the left is more and more, indicating that the nonlinearity of the system is getting stronger.

(5) Considering the influence of current-carrying $i, \mathrm{DC}$ $I$, spacing $b$, wind velocity $U$, tension $T$, and the excitation amplitude $P$ on the nonlinear vibration characteristics of current-carrying iced conductors, it is found that the changes of the above parameters have a certain degree of influence on the nonlinear characteristics of the system such as the response amplitude, the resonance region, and the resonance peak. By changing these parameters, the resonance of the conductors can be enhanced or suppressed. When the system shows the primary resonance and the harmonic resonance, the response amplitude will increase and there will be jump and multivalue phenomena, which will easily affect the safe operation of the conductors. Therefore, the influence of primary resonance and harmonic resonance should be considered when designing structural parameters.

\section{Data Availability}

The data used to support the findings of this study are available from the corresponding author upon request.

\section{Conflicts of Interest}

The authors declare that there are no conflicts of interest regarding the publication of this paper.

\section{Acknowledgments}

This work was financially supported by the National Natural Science Foundation of China (51507106 and 51308570), Cutting Edge Project of Chongqing Science and Technology Commission (cstc2017jcyjAX0246), China Postdoctoral Science Foundation (2020M672238), Chengdu International Science and Technology Cooperation Support Funding (2020-GH02-00059-HZ), Technology Research Project of Chongqing Education Commission (KJ201600712182), and Graduate Research and Innovation Project of Chongqing Jiaotong University (2021S0014).

\section{References}

[1] M. Cai, L. Zhou, H. Lei, and H. Huang, "Wind tunnel test investigation on unsteady aerodynamic coefficients of iced 4bundle conductors," Advances in Civil Engineering, vol. 2019, Article ID 2586242, 12 pages, 2019.

[2] Y. M. Desal, P. Yu, N. Popplewell, and A. H. Shah, "Finite element modeling of transmission line galloping," Computers \& Structures, vol. 57, no. 3, pp. 407-420, 1995.

[3] W. Lou, J. Wang, and Y. Chen, "Effect of motion path of downburst on wind-induced conductor swing in transmission line," Wind and Structures, vol. 23, no. 3, pp. 41-59, 2016.

[4] X. Liu, Y. Hu, and M. Cai, "Free vibration analysis of transmission lines based on the dynamic stiffness method," Royal Society open science, vol. 6, no. 3, pp. 181354-354, 2019.

[5] J. P. D. Hartog, "Transmission line vibration due to sleet," Transactions of the American Institute of Electrical Engineers, vol. 51, no. 4, pp. 1074-1076, 1932.

[6] G. Parkinson, "Phenomena and modeling of flow-induced vibrations of bluff bodies," Progress in Aerospace Sciences, vol. 26, no. 1, pp. 169-224, 1989.

[7] O. Nigol and P. Buchan, "Conductor galloping Part I - den Hartog mechanism," IEEE Transactions on Power Apparatus and Systems, vol. 100, no. 2, pp. 699-707, 1981.

[8] O. Nigol and P. Buchan, "Conductor galloping-Part II torsional mechanism," IEEE Transactions on Power Apparatus and Systems, vol. 100, no. 2, pp. 708-720, 1981, PAS-100. 
[9] P. Yu, Y. M. Desai, A. H. Shah, and N. Popplewell, "ThreeDegree-of-Freedom model for galloping. Part I: formulation," Journal of Engineering Mechanics, vol. 119, no. 12, pp. 2404-2425, 1993.

[10] C. L. Lee and N. C. Perkins, "Nonlinear oscillations of suspended cable containing a two-to-one internal resonance," Nonlinear Dynamics, vol. 3, no. 6, pp. 465-490, 1992.

[11] M. M. Kamel and Y. S. Hamed, "Nonlinear analysis of an elastic cable under harmonic excitation," Acta Mechanica, vol. 214, no. 3, pp. 315-325, 2010.

[12] X. Xie, X. Hu, J. Peng, and Z. Wang, "Refined modeling and free vibration of two-span suspended transmission lines," Acta Mechanica, vol. 228, no. 2, pp. 673-681, 2017.

[13] Z. Wang, H. Kang, C. Sun, Y. Zhao, and Z. Yi, "Modeling and parameter analysis of in-plane dynamics of a suspension bridge with transfer matrix method," Acta Mechanica, vol. 225, no. 12, pp. 3423-3435, 2014.

[14] Y. Zhao, Z. Guo, C. Huang, L. Chen, and S. Li, "Analytical solutions for planar simultaneous resonances of suspended cables involving two external periodic excitations," Acta Mechanica, vol. 229, no. 11, pp. 4393-4411, 2018.

[15] A. Luongo and G. Piccardo, "Non-linear galloping of sagged cables in 1:2 internal resonance," Journal of Sound and Vibration, vol. 214, no. 5, pp. 915-940, 1998.

[16] A. Luongo, D. Zulli, and G. Piccardo, "A linear curved-beam model for the analysis of galloping in suspended cables," Journal of Mechanics of Materials and Structures, vol. 2, no. 4, pp. 675-694, 2007.

[17] A. Luongo, D. Zulli, and G. Piccardo, "Analytical and numerical approaches to nonlinear galloping of internally resonant suspended cables," Journal of Sound and Vibration, vol. 315, no. 3, pp. 375-393, 2008.

[18] Z. Yan, Z. Yan, Z. Li, and T. Tan, "Nonlinear galloping of internally resonant iced transmission lines considering eccentricity," Journal of Sound and Vibration, vol. 331, no. 15, pp. 3599-3616, 2012.

[19] W. Lou, L. Yang, M. F. Huang, and X. Yang, “Two-parameter bifurcation and stability analysis for nonlinear galloping of iced transmission lines," Journal of Engineering Mechanics, vol. 140, no. 11, Article ID 04014081, 2014.

[20] B. Yan, X. Liu, X. Lv, and L. Zhou, "Investigation into galloping characteristics of iced quad bundle conductors," Journal of Vibration and Control, vol. 22, no. 4, pp. 965-987, 2016.

[21] L. Zhou, B. Yan, L. Zhang, and S. Zhou, "Study on galloping behavior of iced eight bundle conductor transmission lines," Journal of Sound and Vibration, vol. 362, pp. 85-110, 2016.

[22] X. Liu, G. Min, M. Cai, B. Yan, and C. Wu, "Two simplified methods for galloping of iced transmission lines," KSCE Journal of Civil Engineering, vol. 25, no. 1, pp. 272-290, 2021.

[23] G. Rega and F. Benedettini, "Non-linear dynamics of an elastic cable under planar excitation," International Journal of NonLinear Mechanics, vol. 22, no. 6, pp. 497-509, 1987.

[24] G. Rega and F. Benedettini, "Planar non-linear oscillations of elastic cables under super-harmonic resonance conditions," International Journal of Non-Linear Mechanics, vol. 132, no. 3, pp. 353-366, 1989.

[25] Y. Zhao, C. Huang, and L. Chen, "Nonlinear planar secondary resonance analyses of suspended cables with thermal effects," Journal of Thermal Stresses, vol. 42, no. 12, pp. 1515-1534, 2019.

[26] Y. Zhao and H. Lin, "Nonlinear dynamics of suspended cables in thermal environments under periodic excitation: two-to- one internal resonance," International Journal of Bifurcation and Chaos, vol. 31, no. 10, 16 pages, Article ID 2150153, 2021.

[27] D. Zulli and A. Luongo, "Bifurcation and stability of a twotower system under wind-induced parametric, external and self-excitation," Journal of Sound and Vibration, vol. 331, no. 2, pp. 365-383, 2012.

[28] A. Luongo and D. Zulli, "Parametric, external and self-excitation of a tower under turbulent wind flow," Journal of Sound and Vibration, vol. 330, no. 13, pp. 3057-3069, 2011.

[29] X. Liu, S. Yang, and C. Wu, "Planar Nonlinear galloping of iced transmission lines under forced self-excitation conditions," Discrete Dynamics in Nature and Society, vol. 12, p. 2021, 2021.

[30] B. Zhang and H. E. Xiao-Xin, "Mechanism simulation for the causes of $220 \mathrm{kV}$ vertical-arrayed double bundle transmission line," Transactions of China Electrotechnical Society, vol. 27, no. 5, pp. 252-259, 2012, in Chinese.

[31] P. Mehta and R. Swart, "Generalized formulation for electromagnetic forces on current-carrying conductors," IEEE Transactions on Power Apparatus and Systems, vol. 86, no. 2, pp. 155-166, 1967.

[32] Y. M. Vasetsky, "Asymptotic method for calculation of electromagnetic fields and forces in systems with spatial conductors configurations," IEEE Transactions on Appiled Superconductivity, vol. 10, no. 1, pp. 1384-1387, 2000.

[33] Y. Xiang, "Analysis of nonlinear torsional vibration for belt driver," Shanghai Journal of Mechanics, vol. 17, no. 4, pp. 339-343, 1996, In Chinese.

[34] C. Touzé, A. Vizzaccaro, and T. Olivier, "Model order reduction methods for geometrically nonlinear structures: a review of nonlinear techniques," Nonlinear Dynamics, vol. 20, pp. 1141-1190, 2021.

[35] CIGRE, "State of the art of conductor galloping," CIGRE, Paris, France, CI-GRE Technical Brochure, No. 322, TF B2. 11, 2007.

[36] Q. Zhang, N. Popplewell, and A. H. Shah, "Galloping of bundle conductor," Journal of Sound and Vibration, vol. 234, no. 1, pp. 115-134, 2000.

[37] Y. Zhao and P. Zheng, "Parameter analyses of suspended cables subjected to simultaneous combination, super and subharmonic excitations," Steel and Composite Structures, vol. 40, no. 2, pp. 203-216, 2021.

[38] X. Liu, G. Min, C. Sun, and M. Cai, "Investigation on stability and galloping characteristics of iced quad bundle conductor," Journal of Applied Fluid Mechanics, vol. 14, no. 1, pp. 117-129, 2021.

[39] H. M. Irvine and T. K. Caughey, "The linear theory of free vibrations of a suspended cable," Proceedings of the Royal Society of London. A. Mathematical and Physical Sciences, vol. 341, no. 1626, pp. 299-315, 1974. 